\title{
RÉGULARITÉ DU PROBLÈME DE KELVIN-HELMHOLTZ POUR L'ÉQUATION D'EULER 2D
}

\author{
Gilles LeBeau ${ }^{1}$
}

\begin{abstract}
We prove that for any solution $u$ locally defined in time of the Kelvin-Helmholtz problem for the Euler $2 \mathrm{~d}$ equation in the plane, then the curve of discontinuity of $u$ and the density of the vortex sheet are analytic (under holder a priori estimates for the curve of discontinuity). We also give a partial result for a solution $u$ defined in a half interval $[O, T[$.
\end{abstract}

Résumé. Nous prouvons que pour toute solution $u$ du problème de Kelvin-Helmholtz des nappes de tourbillons pour l'équation d'Euler bi-dimensionnelle, définie localement en temps, la courbe de saut de $u$ et la densité de tourbillon sont analytiques (sous une hypothèse de régularité Holderienne de la courbe de saut). Nous donnons également un résultat de régularité partielle de la trace de $u$ sur $t=0$ lorsque $u$ est définie sur un demi-interval $[O, T[$.

Classification Mathématique. 35Q05, 35Q23, 35Q24, 35Q35, 35Q57.

Reçu le 14 janvier 2002. Révisé le 12 mars 2002.

\section{INTRODUCTION ET RÉSULtATS}

L'équation d'Euler des fluides parfaits incompressibles

$$
\frac{\partial u}{\partial t}+u \cdot \nabla u=-\nabla p, \operatorname{div}(u)=0
$$

où $u(t, x, y) \in \mathbb{R}^{2}$ est un champ de vecteur dans le plan et où $p(t, x, y) \in \mathbb{R}$ désigne la pression, décrit l'évolution temporelle de la vitesse $u(t, x, y)$ de la particule qui à l'instant $t$ occupe la position $(x, y)$.

Dans (1.1), le terme $u . \nabla$ désigne le champ de vecteur $u_{x} \partial_{x}+u_{y} \partial_{y}$ où $\left(u_{x}, u_{y}\right)$ sont les coordonnées cartésiennes de $u$. La condition d'incompressibilité $\operatorname{div}(u)=0$ permet de réécrire le terme $u$. $\nabla u$ sous la forme

$$
u . \nabla u=\left\{\begin{array}{l}
\partial_{x}\left(u_{x}^{2}\right)+\partial_{y}\left(u_{x} u_{y}\right) \\
\partial_{x}\left(u_{x} u_{y}\right)+\partial_{y}\left(u_{y}^{2}\right)
\end{array}\right.
$$

Mots-clés et phrases : Euler equation, vortex sheets, Kelvin-Helmholtz instability, paradifferential calculus.

${ }^{1}$ Centre de Mathématiques, École Polytechnique, France ; e-mail : lebeau@polytechnique.fr 
ce qui permet de définir des solutions faibles de l'équation d'Euler sous la seule condition de régularité $u \in L_{\mathrm{loc}}^{\infty}\left(t, L_{\mathrm{loc}}^{2}\left(\mathbb{R}^{2}\right)\right)$.

Les quantités formellement conservées pour (1.1) sont

- l'énergie cinétique : $\frac{1}{2} \int_{\mathbb{R}^{2}}|u|^{2} \mathrm{~d} x \mathrm{~d} y$;

- le tourbillon $\omega(t, x, y)=\partial_{x} u_{y}-\partial_{y} u_{x}$ qui est constant le long des trajectoires des particules, i.e. vérifie

$$
\partial_{t} \omega+u . \nabla \omega=0 .
$$

Le problème de Kelvin-Helmholtz des nappes de tourbillon consiste à comprendre la structure des solutions de (1.1) dont la donnée à $t=0, u_{0}(x, y)=u(0, x, y)$ vérifie

$$
\operatorname{rot}\left(u_{0}\right)=\omega_{0}=g_{0}(s) \delta_{\Sigma_{0}} \quad \operatorname{div} u_{0}=0
$$

où $\Sigma_{0}$ est une courbe fermée simple dans le plan, orientée dans le sens direct, paramétrée par l'abscisse curviligne $s, \delta_{\Sigma_{0}}$ désignant la mesure d'intégration sur $\Sigma_{0}$, et où la densité $g_{0}(s)$ vérifie

$$
\exists c_{0}>0 \quad \forall s \quad c_{0} \leq g_{0}(s) \leq 1 / c_{0} .
$$

En notant $u_{0}^{ \pm}$les restrictions de $u$ aux composantes connexes de $\mathbb{R}^{2} \backslash \Sigma$ (le signe + étant associé à la composante non bornée) qui vérifient $\Delta u_{0}^{ \pm}=0$, et par $\left[u_{\perp}\right],\left[u_{/ /}\right]$les sauts des composantes perpendiculaires et tangentielles de $u$ le long de $\Sigma_{0}$ on a

$$
\left[u_{\perp}\right]=0,\left[u_{/ /}\right]=g_{0}=u_{/ /}^{+}-u_{/ /}^{-}
$$

Rappelons deux résultats connus sur ce problème : existence de solutions faibles et persistence de la structure nappes de tourbillons en temps petit si les données sont analytiques.

Théorème 1.1 (Delort 1990). L'équation (1.1) avec donnée de Cauchy $u_{0} \in L^{2}$ tel que $\omega_{0}=\operatorname{rot}\left(u_{0}\right)$ $\in H^{-1} \cap \mathcal{M}_{+}$admet au moins une solution faible ( $\mathcal{M}_{+}$désigne l'espace des mesures positives).

Théorème 1.2 (Bardos et al. 1981). Soit $u_{0}$ vérifiant $\omega_{0}=\operatorname{rot}\left(u_{0}\right)=g_{0}(s) \delta_{\Sigma_{0}}$, où $\Sigma_{0}$ et $g_{0}$ sont analytiques. Alors il existe $T_{*}>0$ tel que (1.1) possède une unique solution $\left.u(t, x, y), t \in\right]-T_{*}, T_{*}\left[, t . q . \operatorname{rot}(u(t,))=.g(t,.) \delta_{\Sigma_{t}}\right.$ avec $\left\{(t, x, y) ;(x, y) \in \Sigma_{t}\right\}$ et $g$ analytiques.

Remarque : Le théorème 2 prouve la conjecture de Garrett-Birkhoff ; on trouvera aussi dans [2] la preuve du résultat analogue en dimension 3 .

Le résultat suivant indique que le problème de Kelvin-Helmholtz est mal posé au sens de Hadamard dans la catégorie des nappes de tourbillons, i.e. ses seules solutions sont celles décrites par le théorème 2.

Théorème 1.3. Soit $\Sigma \subset(] T_{-}, T_{+}\left[\times \mathbb{R}^{2}\right)$ une hypersurface de $\mathbb{R}^{3}$ de la forme $\Sigma=\left\{t,(x, y) \in \Sigma_{t}\right\}$ où $\Sigma_{t}$ est une courbe fermée simple de $\mathbb{R}^{2}$. On suppose $\Sigma$ de classe $C^{1+\rho_{0}}$ pour un $\left.\rho_{0} \in\right] 0,1[$.

Soit $u \in L_{\mathrm{loc}}^{\infty}(]-T, T\left[; L_{\mathrm{loc}}^{2}\left(\mathbb{R}^{2}\right)\right)$ une solution faible de (1.1) vérifiant

$$
\left\{\begin{array}{l}
\operatorname{rot}(u)=\omega(t, x, y)=g(t, s) \delta_{\Sigma_{t}} \\
\lim _{|x, y| \rightarrow \infty} u(t, x, y)=0
\end{array}\right.
$$

où la densité $g$ vérifie

$$
\exists c_{0}>0 \quad \forall(t, s) \quad c_{0} \leq g(t, s) \leq 1 / c_{0} .
$$

Alors $\Sigma$ et g sont analytiques. 
La preuve du théorème 3 s'obtient en étudiant l'équation de Garrett-Birkhoff suivante, qui décrit l'évolution de la courbe $\Sigma_{t}$, paramétrée par $(t, \lambda)$, où $\lambda$ est la densité de tourbillon définie par $g(t, s)=\frac{\partial \lambda}{\partial s}(t, s)$.

$$
\frac{\partial \bar{m}}{\partial t}(t, \lambda)=\frac{1}{2 i \pi} f \frac{\mathrm{d} \lambda^{\prime}}{m(t, \lambda)-m\left(t, \lambda^{\prime}\right)}
$$

Le point clé consiste à vérifier que les hypothèses du théorème 3 entraînent que (1.9) est une équation nonlinéaire elliptique. Il est alors naturel d'attendre également une condition de compatibilité pour les traces en $t=0$ des solutions de $(1.9)$ de classe $C^{1+\rho_{0}}$ sur un intervalle $\left[0, T_{0}\right]$. Le résultat suivant va dans ce sens, mais ne décrit qu'au premier ordre la contrainte que doit satisfaire la donnée initiale en $t=0$ pour qu'il existe une solution classique au problème des nappes de tourbillons dans un petit intervalle $\left[0, T_{0}\right]$.

Théorème 1.4. Soit $\rho_{0}>0$ et $m(t, \lambda) \in C^{1+\rho_{0}}\left(\left[0, T_{0}\right] \times \mathbb{R} / 2 \pi \mathbb{Z} ; \mathbb{C}\right)$ vérifiant l'équation de Garrett-Birkhoff (1.9) sur $] 0, T_{0}[\times \mathbb{R} / 2 \pi \mathbb{Z}$ et

$$
\exists c_{0}>0 \quad \forall t, \lambda, \lambda^{\prime} \quad\left|m(t, \lambda)-m\left(t, \lambda^{\prime}\right)\right| \geq c_{0} \operatorname{dist}\left(\lambda, \lambda^{\prime}\right)
$$

Soit $n(\lambda)=m(0, \lambda)$ la trace de $m$ en $t=0, g(s)=\left(\frac{\mathrm{d} s}{\mathrm{~d} \lambda}\right)^{-1}>0$ la densité de tourbillon en $t=0, s$ désignant l'abscisse curviligne sur la courbe $n$, et $\frac{\mathrm{d} n}{\mathrm{~d} s}=\mathrm{e}^{i \theta(s)}$. On a

$$
\theta(s)+\log g(s) \in C^{\nu}
$$

pour tout $\nu<\nu_{0}=\min \left[1+\rho_{0}, 2 \rho_{0}\right]$.

Remarque : Dans [5] Duchon et Robert ont prouvé que pour toute perturbation assez petite de la droite $y=0$, il existe une densité de tourbillon initiale proche de 1, pour laquelle le problème de Kelvin-Helmholtz possède une solution globalement définie et analytique pour $t \in] 0,+\infty[$. Leur résultat fournit donc (implicitement) une relation de compatibilité complète assurant l'existence dans $t>0$ pour des données proches d'un état constant. Il serait bien sûr très intéressant de pouvoir décrire les relations pseudodifférentielles non-linéaire complètes reliant la courbe de saut et le tourbillon pour assurer l'existence sur des intervalles $\{ \pm t \in] 0, \varepsilon[\}$.

L'article est organisé comme suit. Dans la section 2, nous montrons que le noyau de Garrett-Birkhoff qui intervient dans (1.9) peut s'écrire comme un opérateur de type pseudodifférentiel linéaire agissant sur $m$ modulo un reste plus régulier dans les espaces de Hölder. Pour vérifier ce point, nous utilisons une variante de la méthode de paralinéarisation de Bony [3]. La version $C^{\infty}$ du théorème 1.3 et le théorème 1.4 sont alors obtenus comme conséquence de la théorie elliptique standard dans les sections 3 et 4 . On vérifie l'analyticité de $\Sigma$ et $g$ dans la section 5. Enfin, l'appendice A est consacré à la dérivation de l'équation de Garrett-Birkhoff, et l'appendice B à des rappels de calcul pseudodifférentiel à coefficients peu réguliers. Les notations que nous utiliserons concernant les o.p.d. et les décompositions de Littlewood-Paley sont explicitées dans l'appendice B.

\section{Le noyau de Garrett-Birkhoff}

Nous utiliserons dans cette section les notations et résultats de l'appendice B.

Soit $I=] a, b\left[\right.$ un intervalle de $\mathbb{R}$ et $m(t, x)$ une fonction de classe $C^{1+\rho_{0}}$ sur $I \times \mathbb{R} / 2 \pi \mathbb{Z}$, à valeurs dans $\mathbb{C}$, avec $\rho_{0}>0$ non entier, et vérifiant

$$
\exists c_{0}>0 \quad \forall t, x, x^{\prime} \quad\left|m(t, x)-m\left(t, x^{\prime}\right)\right| \geq c_{0} \operatorname{dist}\left(x, x^{\prime}\right) .
$$


Soit $K(m)(t, x)$ la fonction

$$
K(m)(t, x)=f \frac{\mathrm{d} x^{\prime}}{m(t, x)-m\left(t, x^{\prime}\right)}=\lim _{\varepsilon \rightarrow 0} \int_{\left|x-x^{\prime}\right|>\varepsilon} \frac{\mathrm{d} x^{\prime}}{m(t, x)-m\left(t, x^{\prime}\right)}
$$

qui est bien définie d'après $(2.1)$.

Soit $\left(t_{0}, x_{0}\right) \in I \times \mathbb{R} / 2 \pi \mathbb{Z}$ et $\theta(t, x) \in C_{0}^{\infty}$, à support dans un petit voisinage de $\left(t_{0}, x_{0}\right)$, égal à 1 près de $\left(t_{0}, x_{0}\right)$. L'objet de ce paragraphe est de prouver la

Proposition 2.1. Pour tout $\delta \in] \frac{1}{2}, 1\left[\right.$, on a au voisinage de $\left(t_{0}, x_{0}\right)$

$$
K(m)=\sum_{j=0}^{\infty} S_{j \delta}\left(\frac{-\pi \theta}{\left(\partial_{x} m\right)^{2}}\right) \Delta_{j}\left(\left|D_{x}\right| \theta m\right)+r_{\delta}
$$

avec $r_{\delta} \in C^{\mu}$ près de $\left(t_{0}, x_{0}\right)$, pour tout

$$
\mu<\min \left(2 \delta \rho_{0}, 1+\rho_{0}\right)=\mu_{0} .
$$

Démonstration. Remarquons d'abord que si $Q$ est l'opérateur sur $\mathbb{R}^{2}$ défini par

$$
Q(f)=\sum_{j=0}^{\infty} S_{j \delta}\left(\frac{-\pi \theta}{\left(\partial_{x} m\right)^{2}}\right) \Delta_{j} f
$$

on a $Q \in \Sigma_{\rho_{0}, \delta}^{0}$, et d'après les lemmes B.2 et B.3, $Q\left(\left|D_{x}\right| \theta m\right) \in C^{\rho_{0}} ;$ comme on a supposé $2 \delta>1$, le terme $r$ apparaît comme un reste dans (2.3).

Soit $(\alpha, \beta)=\nabla m\left(t_{0}, x_{0}\right)$; il existe $f \in C_{0}^{1+\rho_{0}}$ à support compact, avec $\left\|f ; C^{1}\right\| \ll 1$, et un voisinage $V$ de $\left(t_{0}, x_{0}\right)$, tels qu'on ait, pour $(t, x) \in V$

$$
m(t, x)=m\left(t_{0}, x_{0}\right)+\alpha\left(t-t_{0}\right)+\beta\left[\left(x-x_{0}\right)+f(t, x)\right] .
$$

Soient $\theta_{1}, \theta_{2} \in C_{0}^{\infty}$ deux fonctions à support dans $V$, égales à 1 près de $\left(t_{0}, x_{0}\right)$, avec $\theta_{2} \equiv 1$ au voisinage du support de $\theta_{1}$. On a

$$
\theta_{1}(t, x) K(m)=f \frac{\theta_{1}(t, x) \theta_{2}\left(t, x^{\prime}\right)}{m(t, x)-m\left(t, x^{\prime}\right)} \mathrm{d} x^{\prime}+f \frac{\theta_{1}(t, x)\left(1-\theta_{2}\left(t, x^{\prime}\right)\right)}{m(t, x)-m\left(t, x^{\prime}\right)} \mathrm{d} x^{\prime} .
$$

Dans (2.7), le deuxième terme du membre de droite appartient à $C^{1+\rho_{0}}$ de sorte qu'en posant

$$
G\left(t, x, x^{\prime}\right)=\frac{\theta_{1}(t, x) \theta_{2}\left(t, x^{\prime}\right)}{\beta\left[1+\frac{f(t, x)-f\left(t, x^{\prime}\right)}{x-x^{\prime}}\right]} \in C^{\rho_{0}}
$$

On a

$$
\theta_{1}(t, x) K(m) \equiv f \frac{G\left(t, x, x^{\prime}\right)}{x-x^{\prime}} \mathrm{d} x^{\prime} \quad\left(\text { modulo } C^{1+\rho_{0}}\right) .
$$

Dans (2.8), on peut remplacer $\frac{f(t, x)-f\left(t, x^{\prime}\right)}{x-x^{\prime}}$ par

$$
u\left(t, x, x^{\prime}\right)=\theta_{3}\left(t, x, x^{\prime}\right) \frac{f(t, x)-f\left(t, x^{\prime}\right)}{x-x^{\prime}} \in C^{\rho_{0}}
$$


avec $\theta_{3} \in C_{0}^{\infty}$ à support proche de $\left(t_{0}, x_{0}, x_{0}\right)$, égal à 1 au voisinage du support de $\theta_{1}(t, x) \theta_{2}\left(t, x^{\prime}\right)$. Pour $\lambda>0$, on décompose alors $u$ sous la forme

$$
u=u_{\lambda}^{1}+u_{\lambda}^{2} ; u_{\lambda}^{1}=S_{\lambda \delta}(u), u_{\lambda}^{2}=u-u_{\lambda}^{1} .
$$

On a alors, uniformément en $\lambda \geq 1$

$$
\left|\partial_{z}^{\alpha} u_{\lambda}^{1}\right|_{\rho_{0}} \leq C_{\alpha}^{t e} 2^{\lambda \delta|\alpha|} ;\left|u_{\lambda}^{2}\right|_{\rho_{0}-\sigma} \leq C_{\sigma}^{t e} 2^{-\lambda \delta \sigma}, 0<\sigma<\rho_{0} .
$$

La formule de Taylor à l'ordre 1 pour $F(\tau)=\frac{1}{1+\tau}$ fournit, avec $z=\left(t, x, x^{\prime}\right)$

$$
\left\{\begin{array}{l}
F(u)=F_{\lambda}^{1}+F_{\lambda}^{2} \\
F_{\lambda}^{1}(z)=F\left(u_{\lambda}^{1}\right)+u_{\lambda}^{2} F^{\prime}\left(u_{\lambda}^{1}\right)=F\left(u_{\lambda}^{1}\right)-u_{\lambda}^{1} F^{\prime}\left(u_{\lambda}^{1}\right)+u F^{\prime}\left(u_{\lambda}^{1}\right) \\
F_{\lambda}^{2}(z)=\left(u_{\lambda}^{2}\right)^{2} \int_{0}^{1} F^{\prime \prime}\left(u_{\lambda}^{1}+t u_{\lambda}^{2}\right)(1-t) \mathrm{d} t
\end{array}\right.
$$

et on a

$$
\begin{gathered}
\left|\partial_{z}^{\alpha} F^{(j)}\left(u_{\lambda}^{1}\right)\right|_{\rho_{0}} \leq C_{j, \alpha}^{t e} 2^{\lambda \delta|\alpha|} \\
\left|F_{\lambda}^{2}\right|_{\rho_{0}-\sigma} \leq C_{\sigma}^{t e} 2^{-2 \lambda \delta \sigma} \text { pour } 0<\sigma<\rho_{0} .
\end{gathered}
$$

Fixons un entier $N_{0}$, et décomposons $\theta_{1} K(m)$ sous la forme

$$
\theta_{1} K(m) \equiv A_{0}+A_{1}+A_{2} \quad\left(\text { modulo } C^{1+\rho_{0}}\right)
$$

avec

$$
\begin{gathered}
A_{0}(t, x)=\sum_{p<N_{0}} \chi_{p}(D)\left[\theta_{1} K(m)\right]+\sum_{p \geq N_{0}} \chi_{p}(D)\left[f \frac{\theta_{1} \theta_{2}}{\beta\left(x-x^{\prime}\right)}\left(F\left(u_{p}^{1}\right)-u_{p}^{1} F^{\prime}\left(u_{p}^{1}\right)\right) \mathrm{d} x^{\prime}\right] \\
A_{1}(t, x)=\sum_{p \geq N_{0}} \chi_{p}(D)\left[f \frac{\theta_{1} \theta_{2}}{\beta\left(x-x^{\prime}\right)} F^{\prime}\left(u_{p}^{1}\right) \frac{f(t, x)-f\left(t, x^{\prime}\right)}{x-x^{\prime}} \mathrm{d} x^{\prime}\right] \\
A_{2}(t, x)=\sum_{p \geq N_{0}} \chi_{p}(D)\left[f \frac{\theta_{1} \theta_{2}}{\beta\left(x-x^{\prime}\right)} F_{p}^{2} \mathrm{~d} x^{\prime}\right]
\end{gathered}
$$

Soit $v_{p}=F\left(u_{p}^{1}\right)-u_{p}^{1} F^{\prime}\left(u_{p}^{1}\right)$. On a pour tout $N,\left|v_{p}\right|_{\rho_{0}+N} \leq C_{N}^{t e} 2^{p \delta N}$. L'opérateur

$$
g\left(t, x, x^{\prime}\right) \mapsto(B g)(t, x)=f \frac{\theta_{1} \theta_{2}}{\left(x-x^{\prime}\right)} g\left(t, x, x^{\prime}\right) \mathrm{d} x^{\prime}
$$

est borne de $C^{\rho_{0}+N}$ dans $C^{\rho_{0}^{\prime}+N}$ pour $\rho_{0}^{\prime}<\rho_{0}$. Les fonctions $w_{p}=f \frac{\theta_{1} \theta_{2}}{\beta\left(x-x^{\prime}\right)} v_{p} \mathrm{~d} x^{\prime}$ vérifient donc $\left|w_{p}\right|_{\rho_{0}^{\prime}+N}$ $\leq C_{N}^{t e} 2^{p \delta N}$, d'où $\left|\chi_{p}(D) w_{p}\right|_{\infty} \leq C_{N}^{t e} 2^{-p\left(\rho_{0}^{\prime}+N\right)} 2^{p \delta N}$. Comme on a $\delta<1$, on en déduit $A_{0} \in C^{\infty}$. 
On a $\left|F_{p}^{2}\right|_{\rho_{0}-\sigma} \leq C_{\sigma}^{t e} 2^{-2 p \delta \sigma}$, donc pour $0<\sigma<\rho_{0}^{\prime}<\rho_{0}$ les fonctions $r_{p}=f \frac{\theta_{1} \theta_{2}}{\beta\left(x-x^{\prime}\right)} F_{p}^{2} \mathrm{~d} x^{\prime}$ vérifient $\left|r_{p}\right|_{\rho_{0}^{\prime}-\sigma} \leq C_{\rho_{0}^{\prime}, \sigma}^{t e} 2^{-2 p \delta \sigma}$. Il en résulte $\left|\chi_{p}(D) r_{p}\right|_{\infty} \leq C_{\rho_{0}^{\prime}}^{t e} 2^{-2 p \delta \rho_{0}^{\prime}}$ pour $\rho_{0}^{\prime}<\rho_{0}$. On a donc $A_{2} \in C^{\mu}$ pour $\mu<2 \delta \rho_{0}$.

On a

$$
A_{1}(t, x)=\sum_{p \geq N_{0}} \chi_{p}(D) f \frac{1}{x-x^{\prime}} g_{p}\left(t, x, x^{\prime}\right) \frac{f(t, x)-f\left(t, x^{\prime}\right)}{x-x^{\prime}} \mathrm{d} x^{\prime}
$$

où $g_{p}\left(t, x, x^{\prime}\right)=\frac{\theta_{1} \theta_{2}}{\beta} F^{\prime}\left(u_{p}^{1}\right)\left(t, x, x^{\prime}\right)$ est à support compact et vérifie $\left|\partial_{z}^{\alpha} g_{p}\right|_{\rho_{0}} \leq C_{\alpha}^{t e} 2^{\delta p|\alpha|}$. Soit $\chi(z) \in \mathcal{S}\left(\mathbb{R}^{3}\right) ;$ tel que $\hat{\chi}(\zeta)$ soit à support dans la boule $\left\{|\zeta| \leq c_{0} \ll 1\right\}$ et égal à 1 près de 0 . On décompose $g_{p}$ sous la forme

$$
g_{p}=a_{p}+b_{p} ; a_{p}(z)=2^{3 p} \int \chi\left(2^{p}\left(z-z^{\prime}\right)\right) g_{p}\left(z^{\prime}\right) \mathrm{d} z^{\prime}
$$

On a alors

$$
\begin{gathered}
\operatorname{support}\left(\widehat{a_{p}}(\zeta)\right) \subset\left\{|\zeta| \leq c_{0} 2^{p}\right\} \\
\left|\partial_{z}^{\alpha} a_{p}\right|_{\rho_{0}} \leq C_{\alpha} 2^{\delta p|\alpha|} \\
\sup _{|z| \geq R_{0}}\left|z^{\alpha} \partial_{z}^{\beta} a_{p}\right|_{L^{\infty}} \in \mathcal{O}_{\alpha, \beta}\left(2^{-p \infty}\right) \quad\left(R_{0} \text { assez grand }\right) \\
\sup _{z}\left|z^{\alpha} \partial_{z}^{\beta} b_{p}\right|_{L^{\infty}} \in \mathcal{O}_{\alpha, \beta}\left(2^{-p \infty}\right) .
\end{gathered}
$$

Les fonctions $u_{p}(z)=\frac{f(t, x)-f\left(t, x^{\prime}\right)}{x-x^{\prime}} b_{p}\left(t, x, x^{\prime}\right)$ vérifient $\left|\left(1+|z|^{2}\right) u_{p}\right|_{\rho_{0}} \in \mathcal{O}\left(2^{-p \infty}\right)$, d'où il résulte

$$
\sum_{p \geq N_{0}} \chi_{p}(D) f \frac{1}{x-x^{\prime}} u_{p}\left(t, x, x^{\prime}\right) \mathrm{d} x^{\prime} \in C^{\infty} .
$$

Pour étudier $\sum_{p \geq N_{0}} \chi_{p}(D) f \frac{1}{x-x^{\prime}} a_{p}\left(t, x, x^{\prime}\right) \frac{f(t, x)-f\left(t, x^{\prime}\right)}{x-x^{\prime}} \mathrm{d} x^{\prime}$, on introduit la décomposition de Littlewood-Paley de $f, f=\sum_{q \geq-1} f_{q}$, avec support $\left(\hat{f}_{q}\right) \subset C_{q}$, et on pose

$$
v_{p, q}=f \frac{1}{x-x^{\prime}} a_{p}\left(t, x, x^{\prime}\right) \frac{f_{q}(t, x)-f_{q}\left(t, x^{\prime}\right)}{x-x^{\prime}} \mathrm{d} x^{\prime} .
$$

On a $\left(\operatorname{avec} \zeta=(\tau, \xi) \in \mathbb{R}^{2}\right)$

$$
\operatorname{support}\left(\widehat{v_{p, q}}(\zeta)\right) \subset\left\{\zeta=\zeta^{\prime}+\zeta^{\prime \prime}, \zeta^{\prime} \in C_{q},\left|\zeta^{\prime \prime}\right| \leq \sqrt{2} c_{0} 2^{p}\right\}
$$

Comme on a $\sqrt{2} c_{0} \ll 1$, il en résulte

$$
\chi_{p}(D)\left(v_{p, q}\right) \equiv 0 \text { pour } q \leq p-2 .
$$


On écrit alors $a_{p}=a_{p}^{1}+a_{p}^{2}$ avec

$$
a_{p}^{1}=a_{p}(t, x, x), a_{p}^{2}(t, x, x+s)=s \int_{0}^{1} \partial_{x^{\prime}} a_{p}(t, x, x+s u) \mathrm{d} u
$$

On a

$$
\left|a_{p}^{2}(t, x, x+s)\right|_{\mu}+\left|\frac{1}{s} a_{p}^{2}(t, x, x+s)\right|_{\mu} \leq C_{\mu}^{k} 2^{p \delta\left[\mu+1-\rho_{0}\right]_{+}} .
$$

En posant $w_{p, q}=\frac{1}{s} a_{p}^{2}(t, x, x+s)\left[f_{q}(t, x)-f_{q}(t, x+s)\right]$ on a donc pour $\mu>0$ petit en utilisant $\left|f_{q}\right|_{\mu}$ $\leq C_{\mu}^{k} 2^{q \mu} 2^{-q\left(1+\rho_{0}\right)}$

$$
\left|s w_{p, q}\right|_{\mu}+\left|w_{p, q}\right|_{\mu} \leq C_{\mu}^{t e}\left[2^{q \mu} 2^{-q\left(1+\rho_{0}\right)} 2^{p \delta\left[1-\rho_{0}\right]_{+}}+2^{-q\left(1+\rho_{0}\right)} 2^{p \delta\left[\mu+1-\rho_{0}\right]_{+}}\right] .
$$

D'où avec $v_{p, q}^{2}=f \frac{1}{s} w_{p, q}(t, x, s) \mathrm{d} s$, pour $0<\varepsilon<\mu$, en utilisant (2.23) pour les grandes valeurs de $s$

$$
\left|v_{p, q}^{2}\right|_{\mu-\varepsilon} \leq C_{\varepsilon, \mu}^{t e}\left[2^{q \mu} 2^{-q\left(1+\rho_{0}\right)} 2^{p \delta\left[1-\rho_{0}\right]_{+}}+2^{-q\left(1+\rho_{0}\right)} 2^{p \delta\left[\mu+1-\rho_{0}\right]_{+}}\right] .
$$

D'où pour $\varepsilon>0$

$$
\left|\sum_{q \geq p-2} \chi_{p}(D) v_{p, q}^{2}\right|_{\infty} \leq C_{\varepsilon} 2^{-p\left(1+\rho_{0}\right)+p \delta\left[1-\rho_{0}\right]_{+}+p \varepsilon}
$$

Il en résulte

On a donc d'après ce qui précède pour $\mu<\mu_{0}$

$$
\sum_{p} \sum_{q \geq p-2} \chi_{p}(D) v_{p, q}^{2} \in C^{\mu} \quad \text { pour } \mu<\mu_{0} .
$$

$$
\theta_{1} K(m) \equiv \sum_{p \geq-1} \chi_{p}(D)\left[a_{p}(t, x, x) \pi\left|D_{x}\right| f\right] \quad\left(\text { modulo } C^{\mu}\right)
$$

puisque

$$
f \frac{f_{q}(t, x)-f_{q}\left(t, x^{\prime}\right)}{\left(x-x^{\prime}\right)^{2}} \mathrm{~d} x^{\prime}=\pi\left|D_{x}\right| f_{q}(t, x)
$$

Pour $|p-q| \leq 2$, on a $\left|a_{p}-a_{q}\right|_{L^{\infty}} \in \mathcal{O}\left(2^{-p \delta \rho_{0}}\right)$. L'opérateur $\Sigma_{p} \chi_{p}(D) a_{p}-a_{p} \chi_{p}(D)=\sum_{|p-q| \leq 2} \chi_{p}\left(a_{p}-a_{q}\right) \chi_{q}$ envoie donc $C^{\rho_{0}}$ dans $C^{\rho_{0}+\delta \rho_{0}}$. Si $\theta \in C_{0}^{\infty}$ vaut 1 près de $\left(t_{0}, x_{0}\right)$, on a donc dans un voisinage $W$ de $\left(t_{0}, x_{0}\right)$, avec $r_{\delta} \in C^{\mu}, \mu<\mu_{0}$.

$$
K(m)=\sum_{p} \pi a_{p}(t, x, x) \chi_{p}(D)\left[\left|D_{x}\right| \theta f\right]+r_{\delta}
$$

(on utilise ici les estimées des Lems. B.2 et B.3 sur les commutateurs $\left[\Sigma a_{p} \chi_{p}, \theta\right]$ et $\left[\left|D_{x}\right|, \theta\right]$ ainsi que $\rho_{0}+1-$ $\left.\delta\left[1-\rho_{0}\right]_{+} \geq \mu_{0}\right)$.

D'après (2.6), si $\theta$ est à support assez petit on a $\theta f \equiv \frac{1}{\beta} \theta m\left(\right.$ modulo $\left.C^{\infty}\right)$ et il reste à vérifier que pour $W$ petit voisinage de $\left(t_{0}, x_{0}\right)$ on a

$$
\left|\frac{a_{p}(t, x, x)}{\beta}+S_{p \delta}\left(\frac{\theta}{\left(\partial_{x} m\right)^{2}}\right)\right|_{L^{\infty}(W)} \in \mathcal{O}\left(2^{-p \delta \rho_{0}}\right) .
$$


D'après $(2.11)$ on a $\left|u_{p}^{1}(z)-u(z)\right|_{\infty} \in \mathcal{O}\left(2^{-p \delta \rho_{0}}\right)$, et d'après $(2.20)\left|a_{p}(z)-g_{p}(z)\right|_{\infty} \in \mathcal{O}\left(2^{-p \delta \rho_{0}}\right)$, donc $\mid \frac{a_{p}(t, x, x)}{\beta}+$ $\left.\frac{1}{\beta^{2}} \frac{1}{\left(1+\left(\partial_{x} f\right)\right)^{2}}\right|_{L^{\infty}(W)} \in \mathcal{O}\left(2^{-p \delta \rho_{0}}\right)$. On a aussi $\left|S_{p \delta}\left(\frac{\theta}{\left(\partial_{x} m\right)^{2}}\right)-\frac{1}{\left(\partial_{x} m\right)^{2}}\right|_{L^{\infty}(w)} \in \mathcal{O}\left(2^{-p \delta \rho_{0}}\right)$ et (2.36) résulte de $\partial_{x} m=\beta\left(1+\partial_{x} f\right)$.

\section{RÉGularité $C^{\infty}$}

Soit $\rho_{0}>0$ et $m(t, x) \in C_{l o c}^{1+\rho_{0}}(] a, b[\times \mathbb{R} / 2 \pi \mathbb{Z}, \mathbb{C})$ vérifiant 2.1 et l'équation de Garrett-Birkhoff.

$$
\frac{\partial}{\partial t} \bar{m}(t, x)=\frac{1}{2 i \pi} f \frac{\mathrm{d} x^{\prime}}{m(t, x)-m\left(t, x^{\prime}\right)} .
$$

L'objet de ce paragraphe est de vérifier le

Théorème 3.1. $m(t, x)$ est $C^{\infty}$ sur $] a, b[\times \mathbb{R} / 2 \pi \mathbb{Z}$.

La preuve va être conséquence de la proposition 2.1 qui entraîne que l'équation (3.1) est une équation non linéaire elliptique.

On conserve les notations de la section 2. D'après (2.3) on a pour tout $\mu<\mu_{0}$

$$
\frac{\partial}{\partial t} \bar{m}-\frac{1}{2 i \pi} Q\left(\left|D_{x}\right| \theta m\right) \in C_{\left(t_{0}, x_{0}\right)}^{\mu} .
$$

On réécrit (3.2) sous la forme d'un système $2 \times 2$ pour $m_{1}=\operatorname{Re}(m), m_{2}=\operatorname{Im}(m)$, la décomposition $Q=Q_{1}+i Q_{2}$ étant associée par (2.5) à la décomposition en parties réelles et imaginaires de $-\pi \theta\left(\partial_{x} m\right)^{-2}$. On obtient

$$
\left(\begin{array}{ll}
A & B \\
C & D
\end{array}\right)\left(\begin{array}{l}
m_{1} \\
m_{2}
\end{array}\right) \in C_{\left(t_{0}, x_{0}\right)}^{\mu}
$$

avec $A=\partial_{t}-\frac{1}{2 \pi} Q_{2}\left(\left|D_{x}\right| \theta\right), B=C=\frac{-1}{2 \pi} Q_{1}\left(\left|D_{x}\right| \theta\right), D=\partial_{t}+\frac{1}{2 \pi} Q_{2}\left(\left|D_{x}\right| \theta\right)$. Les opérateurs A,B,C,D envoient $C_{*}^{r}$ dans $C_{*}^{r-1}$ d'après les résultats de l'appendice $\mathrm{B}$. Soit $\psi \in C_{0}^{\infty}$, égal à 1 près de $\left(t_{0}, x_{0}\right)$, à support petit. D'après les lemmes B.2 et B.3, les commutateurs $[\psi, A], \cdots,[\psi, D]$ envoient $C^{1+\rho_{0}}$ dans $C^{1+\rho_{0}-\nu}$ pour $\nu>\delta\left[1-\rho_{0}\right]_{+}$. On a $1+\rho_{0}-\delta\left[1-\rho_{0}\right]_{+} \geq \mu_{0}$, d'où avec $n_{j}=\psi m_{j} \in C_{0}^{1+\rho_{0}}\left(\mathbb{R}^{2}\right)$

$$
\left(\begin{array}{ll}
A & B \\
C & D
\end{array}\right)\left(\begin{array}{l}
n_{1} \\
n_{2}
\end{array}\right) \in C^{\mu}\left(\mathbb{R}^{2}\right)
$$

En multipliant (3.4) par $\left(\begin{array}{cc}D & -B \\ -C & A\end{array}\right)$, et en réutilisant les lemmes de commutateurs, on obtient (on peut supposer $\theta \psi \equiv \psi$ )

$$
P n_{j} \in C^{\mu-1}\left(\mathbb{R}^{2}\right) ; P=\partial_{t}^{2}-\frac{1}{4 \pi^{2}}\left(Q_{1}^{2}+Q_{2}^{2}\right)\left|D_{x}\right|^{2} .
$$

L'intérêt de (3.5) est qu'on a maintenant $P \in \Sigma_{\rho_{0}, \delta}^{2}$ car $\left|D_{x}\right|^{2}$ est un opérateur différentiel. Or les symboles des opérateurs de $\Sigma_{\rho_{0}, \delta}^{2}$ appartiennent à la classe de Hörmander $S_{1, \delta}^{2}$, et $P$ est elliptique en $\left(t_{0}, x_{0}\right)$ dans $S_{1, \delta}^{2}$ car son symbole est

$$
\sigma(P)=-\tau^{2}-\sum_{j=0}^{\infty} p_{j}(t, x) \chi\left(2^{-j}(\tau, \xi)\right) \xi^{2}
$$

$\operatorname{avec}\left|\partial^{\alpha} p_{j}\right|_{\infty} \leq 2^{\delta j|\alpha|}$ et $\lim _{j \rightarrow \infty} p_{j}(t, x)=\frac{1}{4} \theta^{2}\left|\partial_{x} m\right|^{-4}$. 
Donc (3.5) entraîne $n_{j} \in C_{\left(t_{0}, x_{0}\right)}^{\mu+1}$, c'est-à-dire $m \in C_{\left(t_{0}, x_{0}\right)}^{1+\rho_{0}+\alpha}$ avec $0<\alpha<\mu_{0}-\rho_{0}$. Comme $\left(t_{0}, x_{0}\right)$ est arbitraire, il vient $m \in C_{\mathrm{loc}}^{1+\rho_{0}+\alpha}(] a, b[\times \mathbb{R} / 2 \pi \mathbb{Z})$ donc par itération $m \in C^{\infty}$.

\section{RÉGUlaritÉ DES TRACES}

Soit $\rho_{0}>0$ et $m(t, x) \in C^{1+\rho_{0}}\left(\left[0, T_{0}\right] \times \mathbb{R} / 2 \pi \mathbb{Z} ; \mathbb{C}\right)$ vérifiant (2.1) et l'équation de Garrett-Birkhoff (3.1) sur ] $0, T_{0}[\times \mathbb{R} / 2 \pi \mathbb{Z}$. On pose

$$
n(x)=m(0, x) \in C^{1+\rho_{0}} .
$$

On s'intéresse dans ce paragraphe à décrire au premier ordre l'équation de compatibilité que doit vérifier la trace $n(x)$; cette équation de compatibilité sera obtenue en écrivant les projecteurs de Calderon associés à la paralinéarisation de l'équation de Garrett-Birkhoff.

Dans toute la suite, on notera $\tilde{m}(t, x)$ une extension $C^{1+\rho_{0}}\left(\mathbb{R}_{t} \times \mathbb{R} / 2 \pi \mathbb{Z} ; \mathbb{C}\right)$ de $m$ satisfaisant 2.1 .

Nous utiliserons ici un calcul pseudo différentiel tangentiel : pour $\delta \in] 0,1\left[, \widetilde{\Sigma}_{\rho_{0}, \delta}^{m}\right.$ désignera la classe des o.p.d. tangentiels de symbole $p(t, x, \xi)$ vérifiant

$$
\forall \alpha, \beta, \exists C_{\alpha, \beta} \forall \xi \in \mathbb{R}\left\|\partial_{t, x}^{\alpha} \partial_{\xi}^{\beta} p(., \xi) ; C^{\rho_{0}}\left(\mathbb{R}_{t, x}^{2}\right)\right\| \leq C_{\alpha, \beta}(1+|\xi|)^{m-|\beta|+\delta|\alpha|}
$$

avec la quantification

$$
p\left(t, x, D_{x}\right) f(t, x)=\frac{1}{2 \pi} \int \mathrm{e}^{i x \xi} p(t, x, \xi) \hat{f}(t, \xi) \mathrm{d} \xi
$$

où $\hat{f}(t, \xi)$ désigne la transformée de Fourier partielle.

Nous noterons $\tilde{\phi}(\xi) \in C_{0}^{\infty}(\mathbb{R})$ une fonction paire vérifiant $\tilde{\phi}(\xi) \equiv 1$ pour $|\xi| \leq 1 / c, \tilde{\phi}(\xi)=0$ pour $|\xi| \geq c$, $c \in] 1, \sqrt{2}\left[, \hat{\chi}(\xi)=\tilde{\phi}(\xi / 2)-\tilde{\phi}(\xi), \tilde{S}_{\lambda}(f)(t, x)=\mathcal{F}_{x}^{-1}\left(\tilde{\phi}\left(2^{-\lambda} \xi\right) \hat{f}(t, \xi)\right)\right.$, où $\mathcal{F}_{x}$ et $\hat{f}(t, \xi)$ sont les transformées de Fourier partielles en $x, \tilde{\Delta}_{j}(f)(t, x)=\mathcal{F}_{x}^{-1}\left(\tilde{\chi}\left(2^{-j} \xi\right) \hat{f}(t, \xi)\right), \tilde{\Delta}_{-1}(f)=\tilde{S}_{0}(f)$. La décomposition de LittlewoodPaley partielle s'écrit

$$
f(t, x)=\sum_{j \geq-1}\left(\tilde{\Delta}_{j} f\right)(t, x)
$$

Pour $\mu>0$ non entier et $f \in C_{t, x}^{\mu}$, on a

$$
\left|\tilde{\Delta}_{j} f\right|_{L^{\infty}(t, x)} \leq C 2^{-j \mu}\|f\|_{\mu}
$$

Pour $\mu \in \mathbb{R}$, nous noterons $W^{\mu}$ l'espace des $f(t, x) \in C^{0}\left(\mathbb{R}_{t}, \mathcal{D}_{x}^{\prime}\right)$ vérifiant

$$
\exists A \quad \forall j \quad\left|\tilde{\Delta}_{j} f(t, x)\right|_{L^{\infty}(t, x)} \leq A 2^{-j \mu} .
$$

On a donc l'injection $C_{t, x}^{\mu} \hookrightarrow W^{\mu}$ pour $\mu>0$ non entier. Les opérateurs $P \in \widetilde{\Sigma}_{\rho_{0}, \delta}^{m}$ sont bornés de $W^{\mu}$ dans $W^{\mu-m}$ et les commutateurs $[P, Q], P \in \widetilde{\Sigma}_{\rho_{0}, \delta}^{m^{\prime}}, Q \in \widetilde{\Sigma}_{\rho_{0}, \delta}^{m^{\prime \prime}}$ sont bornés de $W^{\mu}$ dans $W^{\mu-m+1+\nu}$ pour tout $\nu>\delta\left[1-\rho_{0}\right]_{+}$. 
Le résultat de compatibilité sur la trace $n(x)=m(0, x)$ des solutions de l'équation de Garrett-Birkhoff de classe $C^{1+\rho_{0}}$ définies sur $\left[0, T_{0}\right]$ est le suivant :

Théorème 4.1. Pour $\nu<\nu_{0}=\min \left[1+\rho_{0}, 2 \rho_{0}\right]$ on $a$

$$
\operatorname{Im}\left[\log \left(\partial_{x} n\right) \mathrm{e}^{-i \pi / 4}\right] \in C^{\nu}
$$

On notera que (4.7) est indépendant du choix de la détermination du logarithme ; si $s$ est l'absisse curviligne sur la courbe initiale, $g(s)>0$ la densité de tourbillon, et $\frac{\mathrm{d} n}{\mathrm{~d} s}=\mathrm{e}^{i \theta(s)}$, l'équation (4.7) équivaut à la condition du théorème 1.4

$$
\theta(s)+\log g(s) \in C^{\nu} .
$$

Pour vérifier le théorème 4.1, on commence par déduire de la proposition 2.1 dont nous conservons les notations une représentation tangentielle du noyau $K$.

Lemme 4.1. Pour tout $\delta \in] 1 / 2,1\left[\right.$, on a au voisinage de $\left(t_{0}, x_{0}\right)$

$$
K(\tilde{m})=\sum_{j=0}^{\infty} S_{j \delta}\left(\frac{-\pi \theta}{\left(\partial_{x} \tilde{m}\right)^{2}}\right) \tilde{\Delta}_{j}\left(\left|D_{x}\right| \theta m\right)+r_{\delta}
$$

avec $r_{\delta} \in W^{\mu}$ pour tout $\mu<\mu_{0}$.

Démonstration. Dans (4.8), les opérateurs de lissage $S_{j \delta}$ opèrent sur les variables $(t, x)$; la seule différence entre $(2.3)$ et (4.8) est le remplacement de $\Delta_{j}$ par $\tilde{\Delta}_{j}$. Il s'agit donc de vérifier pour $f \in C_{(t, x)}^{\rho_{0}}$

$$
\sum_{j} a_{j}(t, x)\left(\Delta_{j}-\tilde{\Delta}_{j}\right)(f) \in W^{\mu} \text { pour } \mu<\mu_{0}
$$

lorsque les $\left\{a_{j}\right\}$ vérifient

$$
\left\{\begin{array}{l}
\text { spectre }\left(a_{j}\right) \subset\left\{|\tau, \xi| \ll 2^{j}\right\} ;\left|\partial^{\alpha} a_{j}\right|_{\infty} \leq C_{\alpha} 2^{j \delta|\alpha|} \\
\left|a_{j+\ell}-a_{j}\right|_{\infty} \leq C 2^{-\delta \rho_{0} j}(C \text { indépendant de } j, \text { et } \ell \geq 0)
\end{array}\right.
$$

On a en utilisant les conditions de localisation spectrale

$$
\sum_{j} a_{j} \Delta_{j}(f)=\sum_{k, j} \tilde{\Delta}_{k}\left(a_{j} \Delta_{j}(f)\right)=\sum_{j \geq k-2} \tilde{\Delta}_{k}\left(a_{j} \Delta_{j}(f)\right)=\sum_{j \geq k-2} \tilde{\Delta}_{k}\left(a_{k} \Delta_{j}(f)\right)+\sum_{j \geq k-2} \tilde{\Delta}_{k}\left(\left(a_{k}-a_{j}\right) \Delta_{j}(f)\right)
$$

et de même

$$
\sum_{j} a_{j} \tilde{\Delta}_{j}(f)=\sum_{k, j} \tilde{\Delta}_{k}\left(a_{j} \tilde{\Delta}_{j}(f)\right)=\sum_{j \geq k-2} \tilde{\Delta}_{k}\left(a_{k} \tilde{\Delta}_{j}(f)\right)+\sum_{j \geq k-2} \tilde{\Delta}_{k}\left(\left(a_{k}-a_{j}\right) \tilde{\Delta}_{j}(f)\right) .
$$

Pour $j \geq k-2$ et $f \in C_{t, x}^{\rho_{0}}$, on a

$$
\left|\left(a_{k}-a_{j}\right) \Delta_{j}(f)\right|_{\infty}+\left|\left(a_{k}-a_{j}\right) \tilde{\Delta}_{j}(f)\right|_{\infty} \in \mathcal{O}\left(2^{-k \delta \rho_{0}} 2^{-j \rho_{0}}\right) .
$$


Les deux opérateurs $\sum_{j \geq k-2} \tilde{\Delta}_{k}\left(a_{k}-a_{j}\right)\left\{\Delta_{j}, \tilde{\Delta}_{j}\right\}$ envoient donc $C^{\rho_{0}}$ dans $W^{\rho_{0}+\delta \rho_{0}}$. Le lemme résulte alors de

$$
\sum_{j \geq k-2} \tilde{\Delta}_{k}\left(a_{k} \Delta_{j}(f)\right)=\sum_{k} \tilde{\Delta}_{k}\left(a_{k} f\right)=\sum_{j \geq k-2} \tilde{\Delta}_{k}\left(a_{k} \tilde{\Delta}_{j}(f)\right) .
$$

On écrit maintenant l'équation de Garrett-Birkhoff sous forme d'un système tangentiel. Avec $u_{1}=\operatorname{Re}(m)$, $u_{2}=\operatorname{Im}(m)$, on a

$$
\begin{gathered}
u=\left(\begin{array}{l}
u_{1} \\
u_{2}
\end{array}\right) \in C^{1+\rho_{0}}\left(\left[0, T_{0}\right] \times \mathbb{R} / 2 \pi \mathbb{Z} ; \mathbb{R}^{2}\right) \\
\left\{\begin{array}{c}
\partial_{t} u+M\left|D_{x}\right| u=f \in W^{\mu}\left(\left[0, T_{0}\right]\right)\left(\text { pour tout } \mu<\mu_{0}\right) \\
M=\frac{1}{2} \Sigma_{j} S_{j \delta}\left(\begin{array}{cc}
\operatorname{Im}(p) & \operatorname{Re}(p) \\
\operatorname{Re}(p) & -\operatorname{Im}(p)
\end{array}\right) \tilde{\chi}\left(2^{-j} \xi\right) p=\left(\partial_{x} \tilde{m}\right)^{-2} .
\end{array}\right.
\end{gathered}
$$

Dans (4.14), l'opérateur $M$ appartient à la classe $\widetilde{\Sigma}_{\rho_{0}, \delta}^{0}\left(\mathbb{R}_{t} \times \mathbb{R} / 2 \pi \mathbb{Z}\right)$. (On remarquera que si $g(t, x) \in C^{\mu}$ est $2 \pi$ périodique en $x$, les lissages $S_{j \delta}(g)$ sont $2 \pi$-périodiques, et les opérateurs $\tilde{\chi}\left(2^{-j} \xi\right)$ et $\left|D_{x}\right|$ conservent le caractère $2 \pi$ périodique.)

Soient $e_{ \pm}(t, x) \in\left(C^{\rho_{0}}\right)^{2}$ les vecteurs propres normalisés de la matrice

$$
\left(\begin{array}{cc}
\operatorname{Im}(p) & \operatorname{Re}(p) \\
\operatorname{Re}(p) & -\operatorname{Im}(p)
\end{array}\right)
$$

associés aux valeurs propres $\pm|p|(t, x)$ et

$$
\begin{gathered}
Q=\sum_{j} S_{j \delta}\left(\left[e_{+}\right],\left[e_{-}\right]\right) \tilde{\chi}\left(2^{-j} \xi\right) \in \tilde{\Sigma}_{\rho_{0}, \delta}^{0} \\
L=\sum_{j} S_{j \delta}(|p|) \tilde{\chi}\left(2^{-j} \xi\right) \in \tilde{\Sigma}_{\rho_{0}, \delta}^{0} .
\end{gathered}
$$

L'opérateur $Q$ est elliptique ; soit $Q^{-1} \in \tilde{\Sigma}_{\rho}^{0}$ une paramétrix de $Q$ (i.e. $Q \circ Q^{-1}-I d \in \cap_{m} \tilde{\Sigma}_{\rho_{0}, \delta}^{m}, Q^{-1} \circ Q-I d$ $\left.\in \cap_{m} \tilde{\Sigma}_{\rho_{0}, \delta}^{m}\right)$.

Alors $v=Q^{-1} u \in W^{1+\rho_{0}}\left(\left[0, T_{0}\right]\right)$ vérifie,

$$
\begin{gathered}
\partial_{t} v+\left(\begin{array}{cc}
L & 0 \\
0 & -L
\end{array}\right)\left|D_{x}\right| v=g \\
g=\left[\partial_{t}, Q^{-1}\right] u+Q^{-1} M\left|D_{x}\right|\left(Q Q^{-1}-I d\right) u+Q^{-1} f+Q^{-1} M\left[Q,\left|D_{x}\right|\right] v+\left[\left(\begin{array}{cc}
L & 0 \\
0 & -L
\end{array}\right)-Q^{-1} M Q\right]\left|D_{x}\right| v .
\end{gathered}
$$

On a $\left[Q,\left|D_{x}\right|\right] v$ et $\left[\partial_{t}, Q^{-1}\right] u$ dans $W^{\mu}$ pour $\mu<\mu_{0}$. Pour $a \in C^{\rho_{0}}$, on a $\left|S_{j \delta}(a)(t, x)-\tilde{S}_{j-N_{0}}(a(t, .))(x)\right|_{\infty}$ $\in \mathcal{O}\left(2^{-j \delta \rho_{0}}\right)$. En notant $\widetilde{T}_{a}$ le paraproduit tangentiel à $t$ fixé, l'opérateur $\widetilde{T}_{a}-\Sigma S_{j \delta}(a) \tilde{\chi}\left(2^{-j} \xi\right)$ envoie donc $W^{\mu}$ dans $W^{\mu+\delta \rho_{0}}$. 
Comme $\tilde{T}_{a} \circ \tilde{T}_{b}-\tilde{T}_{a b}$ envoie $W^{\mu}$ dans $W^{\mu+\rho_{0}}$, et $\rho_{0}+\delta \rho_{0} \geq \mu_{0}$, on obtient également $\left[\left(\begin{array}{cc}L & 0 \\ 0 & -L\end{array}\right)-Q^{-1} M Q\right]$ $\left|D_{x}\right| v \in W^{\mu}$ pour $\mu<\mu_{0}$. D'où

$$
g \in W^{\mu} \text { pour } \mu<\mu_{0}
$$

Lemme 4.2. Soit $v_{2} \in W^{1+\rho_{0}}\left(\left[0, T_{0}\right]\right)$ solution de

$$
\partial_{t} v_{2}-L\left|D_{x}\right| v=g_{2}
$$

avec $g_{2} \in W^{\mu}$ pour tout $\mu<\mu_{0}$. Alors $v_{2}(0, x) \in C^{\mu+1}$ pour $\mu<\mu_{0}$.

Démonstration. On utilise la méthode de dualité usuelle pour les problèmes aux limites elliptiques. Soit $\tilde{S}_{1, \delta}^{m}$ la classe des opérateurs tangentiels de symbole $a(t, x, \xi)$ vérifiant des estimations

$$
\sup _{t, x}\left|\partial_{x}^{\alpha} \partial_{\xi}^{\beta} a(t, x, \xi)\right| \leq C_{\alpha, \beta}(1+|\xi|)^{m+\delta|\alpha|-|\beta|} .
$$

On a $p(t, x, D)=L\left|D_{x}\right| \in \tilde{S}_{1, \delta}^{1}$, et pour un $c_{0}>0, L(t, x, \xi) \in\left[c_{0}, 1 / c_{0}\right]$ pour $|\xi|$ grand. L'équation

$$
\left\{\begin{array}{l}
\left(\partial_{t} a\right)(t, x, D)+\left({ }^{t} p\right)(t, x, D) \circ a(t, x, D) \in \tilde{S}_{1, \delta}^{-\infty} \\
a(0, x, D)=I d
\end{array}\right.
$$

possède alors une solution $a \in \tilde{S}_{1, \delta}^{0}$ vérifiant de plus

$$
t^{N} a(t, x, D) \in \tilde{S}_{1, \delta}^{-N} \text { pour tout } N
$$

(En utilisant les règles de calcul symbolique, il suffit de vérifier que $a_{0}(t, x, \xi)=\exp -\int_{0}^{t} L(s, x, \xi)|\xi| \mathrm{d} s$ vérifie $t^{N} a_{0} \in \tilde{S}_{1, \delta}^{-N}$ et que si $b(t, x, \xi) \in \cap_{N} t^{-N} \tilde{S}_{1, \delta}^{-m-N}$, on a $\beta(t, x, \xi)=\int_{0}^{t} b(0, x, \xi) \exp \left(-\int_{s}^{t} L(u, x, \xi)|\xi| \mathrm{d} u\right) \mathrm{d} s$ $\in \cap_{N} t^{-N} \tilde{S}_{1, \delta}^{-m-N}$. Ces deux points résultant de

$$
\exp -\int_{0}^{t} L(s, .)|\xi| \mathrm{d} s=\frac{1}{2 i \pi} \int_{\gamma} \frac{\mathrm{e}^{-t|\xi| z}}{z-\alpha} \mathrm{d} z
$$

où $\gamma$ est un contour complexe dans $R e z \geq c_{0} / 2$ entourant $\left[c_{0}, 1 / c_{0}\right]$, et où $\left.\alpha(t, x, \xi)=\int_{0}^{1} L(t u, x, \xi) \mathrm{d} u \in \tilde{S}_{1, \delta}^{0}.\right)$

On fixe alors $x_{0}$ et on pose

$$
h_{k}(t, x)=a(t, x, D) \hat{\tilde{\chi}}_{k}\left(x_{0}-x\right)
$$

où $\hat{\tilde{\chi}}_{k}$ est la $k^{\mathrm{e}}$ troncature de la décomposition de Littlewood-Paley.

Il existe alors $C$ indépendant de $k$ et $x_{0}$ tel que

$$
\int_{0}^{T_{0}} \mathrm{~d} t \int \mathrm{d} x\left|h_{k}(t, x)\right| \leq C 2^{-k}
$$

On peut en effet comme dans la preuve du lemme B.1 décomposer $a$ en $a^{\prime}+a^{\prime \prime}$ où $a^{\prime \prime}(t, x, D)$ envoie $W^{\mu}$ dans $\cap_{\sigma} W^{\sigma}$ et où

$$
a^{\prime}(t, x, D) f=\int \mathrm{d} y \sum_{\ell \geq-1} a_{\ell}^{\prime}(t, x, y) \tilde{\Delta}_{\ell}\left[f\left(t,+x+2^{-\ell} y\right)\right] \mathrm{d} y
$$


Le terme $a^{\prime \prime}(t, x, D)$ fournit une contribution $\mathcal{O}\left(2^{-k \infty}\right)$ à $(4.24)$.

Comme $\tilde{\Delta}_{\ell}\left(\tilde{\chi}_{k}\right)$ n'est non nul que pour $|k-\ell| \leq 2$, on peut remplacer dans (4.24) $h_{k}(t, x)$ par une fonction $e_{k}(t, x)$ vérifiant

$$
\sup _{t} \int\left|t^{N} e_{k}(t, x)\right| \mathrm{d} x \leq C_{N} 2^{-k N}
$$

On a alors

en écrivant $\int_{0}^{T_{0}}=\int_{0}^{2^{-k}}+\int_{2^{-k}}^{T_{0}}$, d'où (4.24).

$$
\int_{0}^{T_{0}} \int\left|e_{k}(t, x)\right| \mathrm{d} t \mathrm{~d} x \leq C^{t e}\left(c_{0}+c_{2}\right) 2^{-k}
$$

En intégrant par parties on obtient

$$
\left\{\begin{array}{l}
\iint_{0}^{T_{0}}\left(\partial_{t} v_{2}-L\left|D_{x}\right| v_{2}\right) h_{k}(t, x) \mathrm{d} t \mathrm{~d} x=\iint_{0}^{T_{0}} g_{2} h_{k} \mathrm{~d} t \mathrm{~d} x= \\
{\left[\int v_{2} h_{k} \mathrm{~d} x\right]_{0}^{T_{0}}-\iint_{0}^{T_{0}} v_{2} b(t, x, D)\left[\hat{\tilde{\chi}}\left(x_{0}-x\right)\right] \mathrm{d} x \mathrm{~d} t}
\end{array}\right.
$$

avec $b=\partial_{t} a+{ }^{t}\left(L\left|D_{x}\right|\right) \circ a \in \tilde{S}_{1, \delta}^{-\infty}$. On a

$$
\left|b(t, x, D)\left[\hat{\tilde{\chi}}_{k}\left(x_{0}-x\right)\right]\right|_{\infty} \leq C_{N} 2^{-k N} \text { et }\left|h_{k}\left(T_{0}, x\right)\right|_{\infty} \leq C_{N} 2^{-k N} .
$$

On a aussi

$$
\int_{0}^{T_{0}} \int g_{2} h_{k} \mathrm{~d} t \mathrm{~d} x=\int_{0}^{T_{0}} \int\left[{ }^{t} a(t, x, D) g_{2}\right] \hat{\tilde{\chi}}_{k}\left(x_{0}-x\right) \mathrm{d} x
$$

et ${ }^{t} a(t, x, D) g_{2}=w(t, x)$ vérifie pour $\mu<\mu_{0}$

$$
\left|t^{N} \tilde{\chi}_{k}(D) w\right|_{\infty} \leq C_{N} 2^{-k(\mu+N)}
$$

d'où comme précédemment pour $\mu<\mu_{0}$

$$
\left|\int_{0}^{T_{0}} \int g_{2} h_{k} \mathrm{~d} t \mathrm{~d} x\right| \leq C 2^{-k(1+\mu)}
$$

Comme $h_{k}(0, x)=\hat{\tilde{\chi}}_{k}\left(x_{0}-x\right)$, on obtient par (4.26) pour $\mu<\mu_{0}$

$$
\left|\tilde{\chi}_{k}(D) v_{2}(0, .)\right|\left(x_{0}\right)=\left|\int h_{k}(0, x) v_{2}(0, x)\right| \leq C 2^{-k(1+\mu)}
$$

d'où le lemme.

Soit $q$ et $\varphi$ les modules et argument de $\partial_{x} \tilde{m}$; on a $p=q^{-2} \mathrm{e}^{-2 i \varphi}$; en identifiant $\mathbb{R}^{2}$ à $\mathbb{C}$, on obtient $e_{ \pm}=\mathrm{e}^{i \varphi} \mathrm{e}^{ \pm i \pi / 4}$, de sorte que la condition $v_{2}(0, x) \in C^{\mu+1}$ se réécrit

$$
\operatorname{Im}\left[\Sigma_{j} S_{j \delta}\left(\left(\partial_{x} \tilde{m}\right)^{-1}\right) \tilde{\chi}\left(2^{-j} \xi\right) \mathrm{e}^{-i \pi / 4} m\right](0, x) \in C^{\mu+1} \quad \forall \mu<\mu_{0} .
$$

Comme $\tilde{m} \in C^{\rho_{0}}$ et $\tilde{m}_{\mid t=0}=n$, on a

$$
\left|S_{j \delta}\left(\left(\partial_{x} \tilde{m}\right)^{-1}\right)(0, x)-\tilde{S}_{j \delta}\left(\left(\partial_{x} n\right)^{-1}\right)(x)\right|_{\infty} \in \mathcal{O}\left(2^{-j \delta \rho_{0}}\right) .
$$


On a aussi $\mid \tilde{S}_{j \delta}\left(\left(\partial_{x} n\right)^{-1}\right)-\tilde{S}_{j-N_{0}}\left(\left(\partial_{x} n\right)^{-1} \mid \in \mathcal{O}\left(2^{-j \delta \rho_{0}}\right)\right.$.

En faisant tendre $\delta$ vers 1 , on peut donc réécrire (4.27) sous forme de paraproduit

$$
\operatorname{Im}\left(\mathrm{e}^{-i \pi / 4} T_{\left(\partial_{x} n\right)^{-1}}(n)\right) \in C^{1+\nu} \quad \forall \nu<\min \left(1+\rho_{0}, 2 \rho_{0}\right)=\nu_{0} .
$$

Comme $\left(\partial_{x} n\right)^{-1} \in C^{\rho_{0}}$ et $n \in C^{1+\rho_{0}}$, on a $T_{\partial_{x}\left(\left(\partial_{x} n\right)^{-1}\right)}(n) \in C^{\nu}$ pour $\nu<\nu_{0}$, d'où en dérivant (4.29)

$$
\operatorname{Im}\left(\mathrm{e}^{-i \pi / 4} T_{\left(\partial_{x} n\right)^{-1}}\left(\left(\partial_{x} n\right)\right)\right) \in C^{\nu} \quad \forall \nu<\nu_{0} .
$$

Or, on a pour $u \in C^{\rho_{0}}$, et $F \in C^{\infty}, F(u) \equiv T_{F^{\prime}(u)} u$ modulo $C^{2 \rho_{0}}$, d'où le théorème 4.1.

\section{RÉgularité analytique}

Soit $m(t, \lambda)$ une solution $C^{\infty}$ de l'équation de Garrett-Birkhoff (1.9). On note $\mathcal{L}$ l'opérateur linéaire

$$
\mathcal{L}(u)=\partial_{t} \bar{u}+\frac{1}{2 i \pi} f \frac{u(t, \lambda)-u\left(t, \lambda^{\prime}\right)}{\left(m(t, \lambda)-m\left(t, \lambda^{\prime}\right)\right)^{2}} \mathrm{~d} \lambda^{\prime} .
$$

On fixe $\rho \in] 0,1\left[\right.$. L'opérateur $\mathcal{L}$ envoie $C_{0}^{1+\rho}(] T_{1}, T_{2}[\times \mathbb{R} / \Lambda \mathbb{Z})$ dans $C_{0}^{\rho}(] T_{1}, T_{2}[\times \mathbb{R} / \Lambda \mathbb{Z})$ et est elliptique donc d'image fermée. Quitte à diminuer $T_{2}-T_{1}$, on peut donc supposer $\mathcal{L}$ injectif, et il existe une constante $M$ telle que

$$
\|u\|_{1+\rho} \leq M\|\mathcal{L} u\|_{\rho} \quad \forall u \in C_{0}^{1+\rho}(] T_{1}, T_{2}[\times \mathbb{R} / \Lambda \mathbb{Z}) .
$$

Notons $g=m(t, \lambda)-m\left(t, \lambda^{\prime}\right), g^{\nu}=\nabla_{t, \lambda}^{\nu} m(t, \lambda)-\nabla_{t, \lambda^{\prime}}^{\nu} m\left(t, \lambda^{\prime}\right)$.

En utilisant la formule

$$
\partial_{x} \int F(f(x)-f(y)) \mathrm{d} y=\int\left(\partial_{x}+\partial_{y}\right) F(f(x)-f(y)) \mathrm{d} y=\int\left(f^{\prime}(x)-f^{\prime}(y)\right) F^{\prime}(f(x)-f(y)) \mathrm{d} y
$$

on obtient en dérivant (1.9)

$$
\mathcal{L}\left(\nabla_{t, \lambda}^{\alpha} m\right)=\frac{1}{2 i \pi} \sum_{\ell=2}^{|\alpha|}(-1)^{\ell} \sum_{\substack { \nu_{1} \\
\begin{subarray}{c}{\left|\cdots+\nu_{\ell}=\alpha\\
\right| \nu_{j} \mid \geq \geq{ \nu _ { 1 } \\
\begin{subarray} { c } { | \cdots + \nu _ { \ell } = \alpha \\
| \nu _ { j } | \geq \geq } }\end{subarray}} \frac{\alpha !}{\nu_{1} ! \cdots \nu_{\ell} !} f \frac{1}{g} \frac{g^{\nu_{1}}}{g} \cdots \frac{g^{\nu_{\ell}}}{g} \mathrm{~d} \lambda^{\prime} .
$$

On peut supposer $\left(T_{1}, T_{2}\right)=(-T, T)$; pour $0<r<r^{\prime}<T$, on choisit des fonctions de localisation $\varphi_{r, r^{\prime}}(t)$ $\in C_{0}^{\infty}(]-r^{\prime}, r^{\prime}[)$ égales à 1 sur $[-r, r]$, telles que l'on ait

$$
\|\varphi(t) f\|_{\rho} \leq C^{t e}\left[\|f\|_{\rho}+\frac{1}{r^{\prime}-r}\|f\|_{\rho-1}\right]
$$

et

On pose pour $k \geq 0$

$$
\left\|\varphi^{\prime}(t) f\right\|_{\rho} \leq C^{t e}\left[\frac{1}{r^{\prime}-r}\|f\|_{\rho}+\frac{1}{\left(r^{\prime}-r\right)^{2}}\|f\|_{\rho-1}\right]
$$

$$
C_{k}(r)=\sum_{|\beta|=k}\left\|\frac{\nabla^{\beta}}{\beta !} m ; C^{1+\rho}([-r, r] \times \mathbb{R} / \Lambda \mathbb{Z})\right\| .
$$

Nous vérifierons le lemme suivant dans l'appendice B. 
Lemme 5.1. On a, avec $h=f(t, \lambda)-f\left(t, \lambda^{\prime}\right)$, quitte à modifier $M$

$$
\left\{\begin{array}{l}
\left\|f \frac{1}{g} \frac{h_{1}}{g} \cdots \frac{h_{\ell}}{g} \mathrm{~d} \lambda^{\prime}\right\|_{\rho} \leq M^{\ell} \prod_{j=1}^{\ell}\left\|f_{j}\right\|_{1+\rho} \\
\left\|f \frac{1}{g} \frac{h_{1}}{g} \cdots \frac{h_{\ell}}{g} \mathrm{~d} \lambda^{\prime}\right\|_{\rho-1} \leq M^{\ell}\left\|f_{1}\right\|_{\rho} \prod_{j=2}^{\ell}\left\|f_{j}\right\|_{1+\rho} .
\end{array}\right.
$$

En utilisant $(5.2,5.4)$ et $\mathcal{L}\left(\varphi \nabla^{\alpha} m\right)=\varphi \mathcal{L}\left(\nabla^{\alpha} m\right)+\varphi^{\prime}(t) \nabla^{\alpha} m$ on obtient pour $0<r<r^{\prime}<T, k \geq 2$

$$
C_{k}(r) \leq M\left[\sum_{\ell=2}^{k} \sum_{\substack{k_{1}+\cdots+k_{\ell}=k \\\left|k_{j}\right| \geq 1}} M^{\ell}\left(1+\frac{1}{k\left(r^{\prime}-r\right)}\right) C_{k_{1}}\left(r^{\prime}\right) \cdots C_{k_{\ell}}\left(r^{\prime}\right)\right]+\frac{M}{r^{\prime}-r} \frac{1}{k} C_{k-1}\left(r^{\prime}\right)+\frac{M}{\left(r^{\prime}-r\right)^{2}} \frac{1}{k^{2}} C_{k-2}\left(r^{\prime}\right) .
$$

En posant $E_{k}=\sup _{r<T} D(T-r)^{\max (k-2,0)} C_{k}(r)$, on obtient en optimisant $(5.5)$ en $\left.r^{\prime} \in\right] r, T[$, pour un $D$ convenable

$$
E_{k} \leq M\left[\sum_{\ell=2}^{k} \sum_{\substack{k_{1}+\cdots, k_{\rho}=k \\ k_{j} \geq 1}} E_{k_{1}} \cdots E_{k_{\ell}}+E_{k-1}+E_{k-2}\right](k \geq 3)
$$

d'où l'existence de $A, B$ tels que

$$
E_{k} \leq A B^{k}
$$

ce qui prouve que $m$ est analytique.

\section{A. L'Équation de Garrett-Birkhoff}

Soit $u$ solution faible de l'équation d'Euler vérifiant les hypothèses du théorème 3 . On note

$$
(t, s) \mapsto(t, M(t, s)) \in \mathbb{R}^{3}
$$

une paramétrisation de l'hypersurface $\Sigma$, avec $M$ de classe $C^{1+\rho_{0}}$ et $\left\|\frac{\partial M}{\partial s}(t, s)\right\| \equiv 1$; la variable $s$ est une abscisse curviligne sur la courbe $\Sigma_{t}$; soit

$$
T(t, s)=\frac{\partial M}{\partial s}(t, s), N(t, s)=R_{\pi / 2}(T(t, s))
$$

les vecteurs tangents et perpendiculaires unitaires à $\Sigma_{t}, R_{\pi / 2}$ désignant la rotation de $\pi / 2$ dans le plan. Les fonctions $T$ et $N$ sont de classe $C^{\rho_{0}}$.

Soit $\psi(t, x, y)$ une fonction de courant telle que

$$
\left(u_{x}, u_{y}\right)=\nabla^{\perp} \psi=\left(-\partial_{y} \psi, \partial_{x} \psi\right)
$$

D'après (1.7), on a $\Delta \psi=g \delta_{\Sigma_{t}}$, d'où, $u$ étant nul à l'infini.

$$
u(t, Q)=R_{\pi / 2} \frac{1}{2 \pi} \int \frac{Q-M(t, s)}{\|Q-M(t, s)\|^{2}} g(t, s) \mathrm{d} s \quad(t, Q) \notin \Sigma .
$$


Soit $u^{+}$(resp. $u^{-}$) la restriction de $u$ à l'ouvert extérieur (resp. intérieur) défini par $\Sigma$. Les champs $u^{ \pm}$ sont harmoniques en $(x, y)$. En utilisant (A.4), on obtient que les traces $\left.u^{ \pm}\right|_{\Sigma}$ existent et sont de la forme $\left.u^{ \pm}\right|_{\Sigma}=A^{ \pm} g+r^{ \pm}$, où $A^{ \pm}(s, \partial s)$ est un o.p.d. de degré zéro en $s$ et où les restes $r^{ \pm}$sont des champs $C^{\rho_{0}-\varepsilon}$ $\operatorname{sur} \Sigma$. On note alors

$$
u_{/ /}^{ \pm}=\left.u^{ \pm}\right|_{\Sigma} \cdot T ; u_{\perp}^{ \pm}=\left.u^{ \pm}\right|_{\Sigma} \cdot N
$$

L'équation $\operatorname{div}(u)=0$ entraîne $u_{\perp}^{+}=u_{\perp}^{-} \stackrel{\text { def }}{=} u_{\perp}$ et on note

$$
\left[u_{/ /}\right]=u_{/ /}^{+}-u_{/ /}^{-} ;\left\langle u_{/ /}\right\rangle=\frac{1}{2}\left(u_{/ /}^{+}+u_{/ /}^{-}\right) .
$$

Soit $v$ le champ de vecteur

$$
v=\left\{\begin{array}{l}
\partial_{x} u_{x}^{2}+\partial_{y} u_{x} u_{y} \\
\partial_{x} u_{x} u_{y}+\partial_{y} u_{y}^{2}
\end{array}\right.
$$

L'équation du tourbillon s'écrit

$$
\partial_{t} \omega+\operatorname{rot}(v)=0
$$

et la distribution $\operatorname{rot}(v)$ est à support dans $\Sigma$. Pour calculer $\operatorname{rot}(v)$, on suppose d'abord $\Sigma$ et $g$ de classe $C^{\infty}$ et on se place dans le système de coordonnées géodésique normal $(s, \ell) \mapsto M(t, s)+\ell N(t, s)$. En notant $\rho(t, s)=\rho$ la courbure de $\Sigma_{t}$ en $s$, on a

$$
\begin{aligned}
& \operatorname{div}(\alpha T+\beta N)=\frac{1}{1-\ell \rho}\left(\frac{\partial \alpha}{\partial s}+\frac{\partial}{\partial \ell}(1-\ell \rho) \beta\right) \\
& \operatorname{rot}(\alpha T+\beta N)=\frac{1}{1-\ell \rho}\left(\frac{\partial \beta}{\partial s}-\frac{\partial}{\partial \ell}((1-\ell \rho) \alpha)\right)
\end{aligned}
$$

et si $u=\alpha T+\beta N$

$$
\left\{\begin{array}{l}
v=a T+b N \\
a=\frac{1}{1-\ell \rho}\left[\partial_{s} \alpha^{2}-2 \rho \alpha \beta\right]+\partial_{\ell}(\alpha \beta) \\
b=\frac{1}{1-\ell \rho}\left[\partial_{s}(\alpha \beta)+\rho\left(\alpha^{2}-\beta^{2}\right)\right]+\partial_{\ell} \beta^{2} .
\end{array}\right.
$$

On en déduit pour $\varphi \in C_{0}^{\infty}\left(\mathbb{R}^{3}\right)$

$$
\langle\operatorname{rot} v, \varphi\rangle=\left.\iint[\alpha] \beta(\nabla \varphi \cdot N)\right|_{\ell=0}-\left.\frac{1}{2} \partial_{s}\left[\alpha^{2}\right] \varphi\right|_{\ell=0} \mathrm{~d} t \mathrm{~d} s
$$

où $[f]=\left.f\right|_{\ell=0}-f_{\mid \ell=0}^{-}$, donc

$$
\langle\operatorname{rot} v, \varphi\rangle=-\iint_{\Sigma}\left[u_{/ /}\right]\left(u_{\perp} \nabla \varphi \cdot N+\left\langle u_{/ /}\right\rangle \frac{\partial \varphi}{\partial s}\right) \mathrm{d} t \mathrm{~d} s
$$

Comme on a $\left[u_{/ /}\right],\left\langle u_{/ /}\right\rangle, u_{\perp} \in L_{\text {loc }}^{\infty}\left(t, \cap_{p} L^{p}\right)$, l'équation (A.10) reste valable sous l'hypothèse $\Sigma \in C^{1+\rho_{0}}, g \in L^{\infty}$. L'équation (A.8) est donc équivalente à

$$
\iint_{\Sigma}\left[u_{/ / /}\right] \mathrm{d} \varphi \cdot\left(\frac{\partial}{\partial t}+u_{\perp} N+\left\langle u_{/ /}\right\rangle T\right) \mathrm{d} t \mathrm{~d} s=0 \quad \forall \varphi \in C_{0}^{\infty}\left(\mathbb{R}^{3}\right)
$$


et (A.11) reste valable pour $\varphi \in C_{0}^{1+\varepsilon}\left(\mathbb{R}^{3}\right)$. Il en résulte déjà

$$
\frac{\partial M}{\partial t} \cdot N=u_{\perp}
$$

donc $u_{\perp} \in C^{\rho_{0}} ;$ comme $A^{ \pm} . N$ est elliptique, on obtient $g \in C^{\rho_{0}-\varepsilon}$, et en réutilisant (A.4)

$$
g=\left[u_{/ /}\right],\left\langle u_{/ /}\right\rangle, u_{\perp} \in C^{\rho_{0}} .
$$

La section $Z=\frac{\partial}{\partial t}+u_{\perp} N+\left\langle u_{/ /}\right\rangle T$ du fibré $\left.T \mathbb{R}^{3}\right|_{\Sigma}$ est tangente à $\Sigma$ et (A.11) s'écrit

$$
\iint g \mathrm{~d} \varphi(Z) \mathrm{d} t \mathrm{~d} s=0 \quad \forall \varphi \in C_{0}^{1}\left(\mathbb{R}^{3}\right)
$$

qui équivaut à

$$
\mathrm{d}(g \mathrm{~d} t \mathrm{~d} s\lrcorner Z)=0
$$

où $\lrcorner$ désigne le produit intérieur. On a $Z=\partial_{t}+\underline{\alpha} \partial_{s}$ avec $\underline{\alpha}=\langle u / /\rangle-\frac{\partial M}{\partial t} \cdot T$ et d'après (A.15), il existe une fonction $\lambda(t, s)$ (sur le revêtement de $\Sigma$ ) telle que

$$
g=\frac{\partial \lambda}{\partial s},-\underline{\alpha} g=\frac{\partial \lambda}{\partial t}
$$

et d'après (A.14) $\int_{\Sigma_{t}} \mathrm{~d} \lambda=\Lambda$ est constant (invariance de la masse totale du tourbillon).

On paramètre alors $\Sigma$ par $\left(t^{\prime}, \lambda\right)$

$$
\left(t^{\prime}, \lambda\right) \in \mathbb{R} \times \mathbb{R} / \Lambda \mapsto m\left(t^{\prime}, \lambda\right)=M(t, s) \quad \lambda=\lambda(t, s), t^{\prime}=t .
$$

On a

$$
u\left(t^{\prime}, Q\right)=R_{\pi / 2} \frac{1}{2 \pi} \int \frac{Q-m\left(t^{\prime}, \lambda\right)}{\left\|Q-m\left(t^{\prime}, \lambda\right)\right\|^{2}} \mathrm{~d} \lambda \quad\left(t^{\prime}, Q\right) \notin \Sigma
$$

et dans le système de coordonnées $\left(t^{\prime}, \lambda\right), Z=\frac{\partial}{\partial t^{\prime}}$.

Comme $\frac{\partial M}{\partial t}=p T+\left[u_{\perp}\right] N$, on obtient

$$
\frac{\partial m}{\partial t^{\prime}}=\frac{\partial M}{\partial t}+\underline{\alpha} T=p T+\left[u_{\perp}\right] N+\left\langle u_{/ /}\right\rangle T-p T=\left\langle u_{/ /}\right\rangle T+\left[u_{\perp}\right] N
$$

d'où d'après (A.17) en notant $f$ la partie finie

$$
\frac{\partial m}{\partial t^{\prime}}\left(t^{\prime}, \lambda\right)=R_{\pi / 2} \frac{1}{2 \pi} f \frac{m\left(t^{\prime}, \lambda\right)-m\left(t^{\prime}, \lambda^{\prime}\right)}{\left\|m\left(t^{\prime}, \lambda\right)-m\left(t^{\prime}, \lambda^{\prime}\right)\right\|^{2}} \mathrm{~d} \lambda^{\prime} .
$$

En identifiant le plan $\mathbb{R}^{2}$ à $\mathbb{C}$ et en notant $\bar{m}$ le complexe conjugué de $m$, on obtient l'équation satisfaite par $\Sigma$, paramétrée dans le système de coordonnées $(t, \lambda)$

$$
\text { Équation de Garrett-Birkhoff }
$$

$$
\frac{\partial \bar{m}}{\partial t}(t, \lambda)=\frac{1}{2 i \pi} f \frac{\mathrm{d} \lambda^{\prime}}{m(t, \lambda)-m\left(t, \lambda^{\prime}\right)}
$$




\section{B. Les espaces $C^{\rho}$ et le CAlCul PSEUdodifférentiel}

Rappelons que pour $\rho \in] 0,1\left[\right.$, l'espace de Hölder $C^{\rho}\left(\mathbb{R}^{d}\right)$ est l'espace des fonctions $u(x)$ bornées telles que la fonction $\frac{u(x)-u(y)}{|x-y|^{\rho}}$ soit bornée sur $\mathbb{R}^{2 d}$, muni de la norme

$$
|u|_{\rho}=\sup |u(x)|+\sup \frac{|u(x)-u(y)|}{|x-y|^{\rho}} .
$$

Pour $\rho=m+r, m \in \mathbb{N}, r \in] 0,1\left[\right.$, l'espace $C^{\rho}\left(\mathbb{R}^{d}\right)$ est l'espace des fonctions $u(x)$ telles que $\partial^{\alpha} u \in C^{r}\left(\mathbb{R}^{d}\right)$ pour tout $\alpha$ tel que $|\alpha| \leq m$, muni de la norme $|u|_{\rho}=\sup _{|\alpha| \leq m}\left|\partial^{\alpha} u\right|_{r}$. Pour $\rho=m+r, m \in-\mathbb{N}$, l'espace $C^{\rho}$ est l'espace des distributions tempérées $u \in \mathcal{S}^{\prime}\left(\mathbb{R}^{d}\right)$ de la forme $u=\sum_{|\alpha| \leq|m|} \partial^{\alpha} u_{\alpha}, u_{\alpha} \in C^{r}$, muni de la norme quotient.

Rappelons la caractérisation par décomposition de Littlewood-Paley des espaces de Hölder $C^{\rho}$. Soit $\left.c \in\right] 1, \sqrt{2}[$ et $\phi(\zeta) \in C_{0}^{\infty}\left(\mathbb{R}^{d}\right)$ une fonction radiale vérifiant $\phi(\zeta) \equiv 1$ pour $|\zeta| \leq 1 / c$ et $\phi(\zeta) \equiv 0$ pour $|\zeta| \geq c$. On pose $\chi(\zeta)=\phi(\zeta / 2)-\phi(\zeta)$ et pour $\lambda \geq 0, f \in \mathcal{S}^{\prime}\left(\mathbb{R}^{d}\right), j \in \mathbb{N}$, en notant $\hat{f}=F(f)$ la transformée de Fourier de $f$

$$
\begin{gathered}
S_{\lambda}(f)=F^{-1}\left(\phi\left(2^{-\lambda} \zeta\right) \hat{f}(\zeta)\right) \\
\Delta_{j}(f)=S_{j+1}(f)-S_{j}(f)=F^{-1}\left(\chi\left(2^{-j} \zeta\right) \hat{f}(\zeta)\right) .
\end{gathered}
$$

Le support de la distribution tempérée $\widehat{\Delta_{j}(f)}$ est contenu dans la couronne

$$
C_{j}=\left\{\zeta,|\zeta| \in\left[2^{j} / c, c 2^{j+1}\right]\right\} .
$$

On a $C_{j+2} \cap C_{j}=\phi$ et la décomposition de Littlewood-Paley de $f \in \mathcal{S}^{\prime}$ est

$$
f=S_{0}(f)+\sum_{j=0}^{\infty} \Delta_{j}(f)
$$

Les espaces de Hölder $C^{\rho}$ sont caractérisés comme suit. Pour $\rho \in \mathbb{R} \backslash \mathbb{Z}$, il existe une constante $A$ telle que

$$
\forall f \in C^{\rho} \quad \Delta_{j}(f) \in L^{\infty} \text { et } \sup _{j \geq-1}\left|\Delta_{j}(f)\right|_{L^{\infty}} 2^{j \rho} \leq A|f|_{\rho}
$$

où on a noté $S_{0}(f)=\Delta_{-1}(f)$. Réciproquement, si $\left\{u_{j}\right\}_{j \geq-1}$ est une suite de fonctions bornées vérifiant support $\left(\hat{u}_{j}\right) \subset C_{j}$ (où $C_{-1}$ désigne la boule $\{|\zeta| \leq c\}$ ) et $\left|u_{j}\right|_{L^{\infty}} \leq M 2^{-j \rho}$ pour tout $j$, alors la série $\Sigma u_{j}$ est convergente dans $\mathcal{S}^{\prime}$, sa somme $u$ appartient à $C^{\rho}$ et on a $|u|_{\rho} \leq A \sup _{j \geq-1} 2^{j \rho}\left|u_{j}\right|_{L^{\infty}}$.

Nous utiliserons le calcul pseudodifférentiel suivant sur $\mathbb{R}^{d}$. Pour $\left.\delta \in\right] 0,1\left[, \rho_{0} \in \mathbb{R} \backslash \mathbb{Z}, m \in \mathbb{R}\right.$ nous noterons $\sum_{\rho_{0}, \delta}^{m}$ la classe des opérateurs pseudo différentiels de symbole $p(z, \zeta)$ vérifiant

$$
\forall \alpha, \beta \quad \exists C_{\alpha, \beta} \quad \forall \zeta \quad\left\|\partial_{z}^{\alpha} \partial_{\zeta}^{\beta} p(., \zeta) ; C^{\rho_{0}}\left(\mathbb{R}_{z}^{d}\right)\right\| \leq C_{\alpha, \beta}(1+|\zeta|)^{m-|\beta|+\delta|\alpha|}
$$

avec la quantification

$$
p(z, D) f=(2 \pi)^{-d} \int \mathrm{e}^{i z \zeta} p(z, \zeta) \hat{f}(\zeta) \mathrm{d} \zeta
$$


Lemme B.1. Soit $P=p(z, D)$ dans la classe $\Sigma_{\rho_{0}, \delta}^{m}$. On a $P=P^{\prime}+P^{\prime \prime}$, où l'opérateur $P^{\prime \prime}$ envoie $C^{\rho}$ dans $\cap C^{r}$ pour tout $\rho$, et où $P^{\prime} \in \Sigma_{\rho_{0}, \delta}^{m}$ est de la forme

$$
P^{\prime}(f)=\int_{\mathbb{R}^{d}} \sum_{k \geq-1} p_{k}^{\prime}(z, y) \Delta_{k}\left(f\left(z+2^{-k} y\right)\right) \mathrm{d} y
$$

où la suite de fonctions $p_{k}^{\prime}(z, y) \in C^{\infty}\left(\mathbb{R}^{2 d}\right)$ vérifie

i) le support de la transformée de Fourier $\widehat{p_{k}^{\prime}}(\zeta, y)$

$$
\text { est contenu dans la boule }\left\{|\zeta| \leq \frac{c}{4} 2^{k}\right\}
$$

ii) pour tout $\alpha, \beta, \gamma$ il existe $C_{\alpha, \beta, \gamma}$ tels que

Démonstration. Le symbole de $P^{\prime}$ est

$$
\forall k, \forall y \quad\left|\partial_{z}^{\alpha} y^{\beta} \partial_{y}^{\gamma} p_{k}^{\prime}(., y)\right|_{\rho_{0}} \leq C_{\alpha, \beta, \gamma} 2^{\delta|\alpha| k} 2^{k m}
$$

$$
p^{\prime}(z, \zeta)=\sum_{k \geq-1} \int_{\mathbb{R}^{d}} p_{k}^{\prime}(z, y) \mathrm{e}^{i y 2^{-k} \zeta} \chi_{k}(\zeta) \mathrm{d} y
$$

avec $\chi_{k}(\zeta)=\chi\left(2^{-k} \zeta\right)$ pour $k \geq 0$ et $\chi_{-1}(\zeta)=\phi(\zeta)$, de sorte que $p^{\prime}(z, \zeta)$ vérifie les estimations (B.7) d'après (B.11).

Réciproquement, si $p(z, \zeta)$ vérifie (B.7), il existe des $p_{k}$ vérifiant (B.11) tels qu'on ait

$$
p(z, \zeta)=\sum_{k \geq-1} \int_{\mathbb{R}^{d}} p_{k}(z, y) \mathrm{e}^{i y 2^{-k} \zeta} \chi_{k}(\zeta) \mathrm{d} y
$$

Il suffit pour cela de choisir deux fonctions $\theta_{-1}(\zeta) \in C_{0}^{\infty}\left(\mathbb{R}^{d}\right), \theta(\zeta) \in C_{0}^{\infty}\left(\mathbb{R}^{d} \backslash 0\right)$ vérifiant $\theta_{-1}(2 \zeta) \equiv 1$ au voisinage du support de $\phi=\chi_{-1}$ et $\theta(\xi) \equiv 1$ au voisinage du support de $\chi$, et poser

(B.13) résulte alors de

$$
\begin{gathered}
p_{-1}(z, y)=(2 \pi)^{-d} \int p(z, u / 2) \theta_{-1}(u) \mathrm{e}^{-i u y} \mathrm{~d} u \\
p_{k}(z, y)=(2 \pi)^{-d} \int p\left(z, 2^{k} u\right) \theta(u) \mathrm{e}^{-i u y} \mathrm{~d} u
\end{gathered}
$$

$$
p(z, \zeta)=\sum_{k \geq-1} p(z, \zeta) \chi_{k}(\zeta)=p(z, \zeta) \theta_{-1}(2 \zeta) \phi(\zeta)+\sum_{k \geq 0} p(z, \zeta) \theta\left(2^{-k} \zeta\right) \chi_{k}(\zeta)
$$

Or toute suite de fonctions $C^{\infty} p_{k}(z, y)$ vérifiant (B.11) se décompose sous la forme $p_{k}=p_{k}^{\prime}+p_{k}^{\prime \prime}$, où $p_{k}^{\prime}$ vérifie (B.11) et $(\mathrm{B} .10)$ et où $p_{k}^{\prime \prime}(z, y)$ vérifie

Pour tout $\alpha, \beta, \gamma, N$, il existe $C_{\alpha, \beta, \gamma, N}$ tels que

$$
\forall k, \quad\left|\partial_{z}^{\alpha} y^{\beta} \partial_{y}^{\gamma} p_{k}^{\prime \prime}(., y)\right|_{L_{(z, y)}^{\infty}} \leq C_{\alpha, \beta, \gamma N} 2^{-N k}
$$


Il suffit pour cela de poser $p_{k}^{\prime}=S_{k-2} p_{k}$; les opérateurs $S_{\lambda}$ étant uniformément bornées en $\lambda$ sur $C^{\rho_{0}}$ et commutant aux $\partial_{z}^{\alpha}, p_{k}^{\prime}$ vérifie bien (B.10) et (B.11). On a aussi

$$
\left\{\begin{array}{l}
\forall N, \beta, \gamma \quad \exists C_{N, \beta, \gamma}, \forall_{j} \geq 0 \quad \forall k \quad \forall y \\
\left\|2^{2 j N} \chi_{j}\left(D_{z}\right) y^{\beta} \partial_{y}^{\gamma} p_{k}(. y)\right\|_{L^{\infty}(z)} \leq C_{N, \beta, \gamma} 2^{-j \rho_{0}} 2^{2 N k \delta} 2^{k m}
\end{array}\right.
$$

(Si $\hat{\psi}(\zeta) \in C_{0}^{\infty}\left(\mathbb{R}^{d} \backslash 0\right)$ est égal à 1 près de la couronne $C_{0}$, et $\hat{\psi}_{N}(\zeta)=\left(\frac{-1}{\left.\zeta\right|^{2}}\right)^{N} \hat{\psi}(\zeta) \in C_{0}^{\infty}\left(\mathbb{R}^{d} \backslash 0\right)$, on a $2^{2 j N} \chi_{j}\left(D_{z}\right) p_{k}=2^{2 j N} \hat{\psi}\left(2^{-j} \zeta\right) \chi_{j}\left(D_{z}\right) p_{k}=\hat{\psi}_{N}\left(2^{-j} \zeta\right) \chi_{j}\left(D_{z}\right) \Delta_{z}^{N} p_{k}$; comme $\hat{\psi}_{N}\left(2^{-j} \zeta\right)$ est borné uniformément en $j$ sur $L^{\infty}$ (B.15) résulte de (B.11) et (B.6).)

Comme $p_{k}^{\prime \prime}(z, y)=\sum_{j \geq k-2} \chi_{j}\left(D_{z}\right) p_{k}(z, y)$, l'équation (B.14) résulte de (B.15) et de $\delta<1$.

Comme, pour $f \in C^{\rho}$, on a $\left|\partial_{z}^{\alpha} \Delta_{k} f\left(z+2^{-k} y\right)\right|_{L_{(z, y)}^{\infty}} \leq C_{\alpha} 2^{k|\alpha|} 2^{-k \rho}|f|_{\rho}$ l'opérateur $P^{\prime \prime}$, défini par (B.9) avec $p_{k}^{\prime \prime}$, vérifie donc

$$
\forall \alpha, \exists C_{\alpha} \quad\left|\partial_{z}^{\alpha} P^{\prime \prime}(f)\right|_{L^{\infty}} \leq C_{\alpha}|f|_{\rho}
$$

donc est borné de $C^{\rho}$ dans $C^{r}$ pour tout couple $(\rho, r)$.

Il résulte du lemme précédent que pour $\rho_{0}>0$, un opérateur $P(z, D)$ dans la classe $\Sigma_{\rho_{0}, \delta}^{m}$ est borné de $C_{*}^{r}$ dans $C_{*}^{r-m}$ pour tout $r \in \mathbb{R}$, où $C_{*}^{r}$ est l'espace de Besov (qui coïncide avec $C^{r}$ pour $r$ non entier)

$$
C_{*}^{r}=\left\{f \in \mathcal{S}^{\prime}, \forall_{j} \geq-1, \Delta_{j}(f) \in L^{\infty} \text { et } \sup _{j \geq-1}\left|\Delta_{j}(f)\right|_{L^{\infty}} 2^{j \rho}<+\infty\right\}
$$

On a en effet $p^{\prime}(f)=\sum_{k \geq-1} g_{k}$, où $g_{k}(z)=\int_{\mathbb{R}^{d}} p_{k}^{\prime}(z, y) \Delta_{k}\left(f\left(z+2^{-k} y\right) \mathrm{d} y\right.$ vérifie support $\left(\hat{g}_{k}(\zeta)\right) \subset C_{k}^{\prime}$, $C_{k}^{\prime}=\left\{|\zeta| \in\left[2^{k}\left(\frac{1}{c}-c / 4\right), 2^{k}(2 c+c / 4)\right]\right.$ pour $k \geq 0, C_{-1}^{\prime}=\{|\zeta| \leq c+c / 4\} ;$ on a $C_{k}^{\prime} \subset C_{k-1} \cup C_{k} \cup C_{k+1}$, donc $\left|\Delta_{j}\left(p^{\prime}(f)\right)\right|_{\infty} \leq C^{t e} \sum_{|k-j| \leq 1}\left|g_{k}\right|_{\infty} \leq C^{t e} 2^{j m} 2^{-j r}|f|_{C_{*}^{r}}, \operatorname{car}\left|\Delta_{k}\left(f\left(z+2^{-k} y\right)\right)\right|_{\infty}=\left|\Delta_{k}(f)\right|_{\infty}$

Comme $C^{\rho_{0}}$ est une algèbre pour $\rho_{0}>0$, la formule de composition des opérateurs pseudodifférentiels,

$$
\begin{gathered}
p(z, D) \circ q(z, D)=r(z . D) \\
r(z, \zeta)=(2 \pi)^{-d} \int \mathrm{e}^{-i x \theta} p(z, \zeta+\theta) q(z+x, \zeta) \mathrm{d} x \mathrm{~d} \theta \\
r(z, \zeta)=\sum_{|\alpha| \leq N} \frac{1}{i^{|\alpha|} \alpha !} \partial_{\zeta}^{\alpha} p(z, \zeta) \partial_{z}^{\alpha} q(z, \zeta)+r_{N+1}(z, \zeta) \\
r_{N+1}(z, \zeta)=\sum_{|\gamma|=N+1}(2 \pi)^{-d} \int_{0}^{1}(1-t)^{N+1} \mathrm{~d} t \int \mathrm{e}^{-i x \theta} \frac{\theta^{\gamma}}{\gamma !} \partial_{\zeta}^{\gamma} p(z, \zeta+t \theta) q(z+x, \zeta) \mathrm{d} x \mathrm{~d} \theta
\end{gathered}
$$

implique qu'on a pour $P \in \Sigma_{\rho_{0}, \delta}^{m}$ et $Q \in \Sigma_{\rho_{0}, \delta}^{m^{\prime}}, P \circ Q \in \Sigma_{\rho_{0}, \delta}^{m+m^{\prime}}$ et pour tout $N$

$$
P \circ Q-\sum_{|\alpha| \leq N} \frac{1}{i^{|\alpha|} \alpha !} \partial_{\zeta}^{\alpha} p \partial_{z}^{\alpha} q(z, D) \in \Sigma_{\rho_{0}}^{m+m^{\prime}-(N+1)(1-\delta)} .
$$


$\left(\operatorname{Car} \partial_{\zeta}^{\alpha} p \partial_{z}^{\alpha} q \in \Sigma_{\rho_{0}, \delta}^{m+m^{\prime}-|\alpha|(1-\delta)}\right.$, et $\partial_{z}^{\alpha} \partial_{\zeta}^{\beta} r_{N+1}$ est combinaison linéaire finie de termes de la forme

$$
J(x, \zeta)=\int_{0}^{1}(1-t)^{N+1} \mathrm{~d} t \int \mathrm{e}^{-i x \theta} \partial_{\zeta}^{\gamma+\beta_{1}} \partial_{z}^{\alpha_{1}} p(z, \zeta+t \theta) \partial_{\zeta}^{\beta_{2}} \partial_{z}^{\gamma+\alpha_{2}} q(z+x, \zeta) \mathrm{d} x \mathrm{~d} \theta
$$

où l'intégrale en $(x, \theta)$ est oscillante. En utilisant $\delta<1,\left(1-\Delta_{\theta}\right)^{N} \mathrm{e}^{-i x \theta}=\left(1+x^{2}\right)^{N} \mathrm{e}^{-i x \theta},\left(1-\Delta_{x}\right)^{M} \mathrm{e}^{-i x \theta}$ $=\left(1+\theta^{2}\right)^{M} \mathrm{e}^{-i x \theta},(1+|\zeta+t \theta|)^{\nu} \leq C(1+|\zeta|)^{\nu}(1+|\theta|)^{|\nu|}$, on obtient en intégrant par parties dans l'intégrale définissant $J(z, \zeta)$, la propriété :

Pour tout $A>0$ et tout $p$, il existe $N(p, A)$ tel que $|\alpha|+|\beta| \leq p$ et $N \geq N(p, A)$ implique

$$
\| \partial_{z}^{\alpha} \partial_{\zeta}^{\beta} r_{N+1}(\cdot, \zeta) ; C^{\rho_{0}}\left(\mathbb{R}_{\zeta}^{d} \| \leq C^{t e}(1+|\zeta|)^{-A}\right.
$$

ce qui prouve (B.19).)

Lemme B.2. Soit $P \in \Sigma_{\rho_{0}, \delta}^{m^{\prime}}, Q \in \Sigma_{\rho_{0}, \delta}^{m^{\prime \prime}}, m=m^{\prime}+m^{\prime \prime}$

i) pour $\left.\rho_{0} \in\right] 0,1\left[\right.$, le commutateur $[P, Q]$ est borné de $C_{*}^{r}$ dans $C_{*}^{r-m+1-\nu}$ pour tout $r$ et tout $\nu>\delta\left(1-\rho_{0}\right)$;

ii) pour $\rho_{0}>1$, le commutateur $[P, Q]$ est borné de $C_{*}^{r}$ dans $C_{*}^{r-m+1}$ pour tout $r$.

Démonstration. D'après (B.19), il suffit d'étudier l'opérance des opérateurs $\left(\partial_{\zeta}^{\gamma} p \partial_{\xi}^{\gamma} q\right)(z, D)=c_{\gamma}(z, D)$ pour $|\gamma| \geq 1$.

Pour $\rho_{0}>1$, on a $c_{\gamma}(z, \zeta) \in \Sigma_{\rho_{0}-1, \delta}^{m-1}$, d'où ii).

Pour $\left.\rho_{0} \in\right] 0,1\left[\right.$ et $0<t<\rho_{0}$ on a $\partial_{\zeta}^{\gamma} p \in \Sigma_{\rho_{0}-t, \delta}^{m^{\prime}-|\gamma|}$ et (en utilisant l'inégalité de convexité $|f|_{\alpha \rho_{1}+(1-\alpha) \rho_{2}}$ $\left.\leq C|f|_{\rho_{1}}^{\alpha}|f|_{\rho_{2}}^{1-\alpha}\right) \partial_{z}^{\gamma} q \in \Sigma_{\rho_{0}-t, \delta}^{m^{\prime \prime}+\delta|\gamma|-t \delta}$, donc $c_{\gamma} \in \Sigma_{\rho_{0}-t, \delta}^{m-1+\delta(1-t)}$ d'où i) en faisant tendre $t$ vers $\rho_{0}$.

Si $z=\left(z^{\prime}, z^{\prime \prime}\right), z^{\prime} \in \mathbb{R}^{d^{\prime}}, z^{\prime \prime} \in \mathbb{R}^{d^{\prime \prime}}$, nous utiliserons également des résultats d'opérance de l'opérateur $\left|D_{z^{\prime}}\right|$ défini par

$$
\left|D_{z^{\prime}}\right| u=K * u, \widehat{K}(\zeta)=\left|\zeta^{\prime}\right| \quad \zeta=\left(\zeta^{\prime}, \zeta^{\prime \prime}\right)
$$

On a $K=K^{\prime}\left(z^{\prime}\right) \otimes \delta_{z^{\prime \prime}=0}$, où la distribution $K^{\prime}$ est invariante par rotation, homogène de degré $-1-d^{\prime}$, donc égale à $C^{t e} p f\left|z^{\prime}\right|^{-\left(1+d^{\prime}\right)}$.

Lemme B.3. i) $\left|D_{z^{\prime}}\right|$ est borné de $C_{*}^{r}$ dans $C_{*}^{r-1}$ pour tout $r$.

ii) Pour $\rho_{0}>0$ et $p(z, D) \in \Sigma_{\rho_{0}, \delta}^{0}$, le commutateur $\left[P,\left|D_{z^{\prime}}\right|\right]$ est borné de $C_{*}^{r}$ dans $C_{*}^{r-\nu}$ pour tout $\nu>\delta\left[1-\rho_{0}\right]_{+}$.

\section{Démonstration.}

i) On peut décomposer $K^{\prime}$ sous la forme $K^{\prime}=K_{0}^{\prime}+\ell\left(x^{\prime}\right)$ où $K_{0}^{\prime} \in \mathcal{E}^{\prime}$ est à support dans $\left|z^{\prime}\right| \leq 1$ et $\ell \in L^{1}\left(\mathbb{R}^{d^{\prime}}\right)$. Soit $u \in C_{*}^{r}$ et $u=\sum_{-i}^{\infty} u_{j}$ la décomposition de Littlewood-Paley de $u$; on a $\left|u_{j}\right|_{\infty} \leq C|u|_{c_{*}^{r}} 2^{-j r}$. La distribution tempérée $v_{j}=\left(K_{0}^{\prime} \otimes \delta_{z^{\prime \prime}=0}\right) * u_{j}+\left(k \otimes \delta_{z^{\prime \prime}=0}\right) * u_{j}$ est bien définie et le support de $\hat{v}_{j}$ est contenu dans la couronne $C_{j}$. Soit $\psi \in C_{0}^{\infty}\left(\mathbb{R}^{d}\right)$ tel que $\psi(\zeta) \equiv 1$ pour $|\zeta| \leq 4 c$. On a $\psi\left(2^{-j} D\right) u_{j}=u_{j}$. Il suffit donc de vérifier qu'on a

$$
K * 2^{j d} \hat{\psi}\left(2^{j} z\right)=A_{j} \in L^{1}\left(\mathbb{R}^{d}\right) \text { et }\left\|A_{j}\right\|_{L^{1}} \leq C 2^{j} .
$$

La fonction $\widehat{K}$ étant homogène de degré 1 , on a $A_{j}(z)=2^{j(d+1)} A_{0}\left(2^{j} z\right)$ et $A_{0}=A_{0}^{1}+A_{0}^{2}$, avec

$$
A_{0}^{1}=\left(K_{0}^{\prime} \otimes \delta_{z^{\prime \prime}=0}\right) * \hat{\psi} \in \mathcal{S}\left(\mathbb{R}^{d}\right) \text { et } A_{0}^{2}=\left(\ell \otimes \delta_{z^{\prime \prime}=0}\right) * \hat{\psi} \in L^{1},
$$

d'où le résultat. 
ii) On peut supposer $P$ de la forme (B.9). Pour $f \in C_{*}^{r}$, on a

$$
\Delta_{k}\left(\left|D_{z^{\prime}}\right| f\right)\left(z+2^{-k} y\right)=\left(\left|D_{z}\right|^{\prime} \Delta_{k}(f)\right)\left(z+2^{-k} y\right)
$$

et la fonction $v_{k}(z, y)=\left(\Delta_{k}(f)\right)\left(z+2^{-k} y\right)$ vérifie $\left|v_{k}\right|_{\infty} \leq C^{t e}|f|_{C_{*}^{r}} 2^{-r k}$. On a

$$
\left\{\begin{aligned}
{\left[D_{z^{\prime}}, P\right] f } & =\int_{\mathbb{R}^{d}} \mathrm{~d} y \sum_{k \geq-1} g_{k}(z, y) \\
g_{k}(z, y) & =\left|D_{z^{\prime}}\right| p_{k}(z, y) v_{k}-p_{k}(z, y)\left|D_{z^{\prime}}\right| v_{k} .
\end{aligned}\right.
$$

Comme le spectre de $g_{k}$ est contenu dans $C_{k}^{\prime}$, il suffit de vérifier $\left|g_{k}(., y)\right|_{\infty} \leq C^{t e}|f|_{C_{*}^{r}} 2^{-\rho k}(1+|y|)^{-(1+d)}$ pour $\rho<r-\delta\left[1-\rho_{0}\right]_{+}$.

D'après le point i) on a

$$
g_{k}(z, y)=\int A_{k}(z-w)\left[p_{k}(w, y)-p_{k}(z, y)\right] v_{k}(w, y) \mathrm{d} w .
$$

Il suffit donc de vérifier qu'on a, pour $\nu>\delta\left[1-\rho_{0}\right]_{+}$

$$
\int\left|A_{k}(z-w) \| p_{k}(w, y)-p_{k}(z, y)\right| \mathrm{d} w \leq C_{\nu} 2^{k \nu}(1+|y|)^{-(1+d)} .
$$

D'après (B.11), on a

$$
\left\{\begin{array}{l}
\left|p_{k}(w, y)-p_{k}(z, y)\right| \leq c_{\beta}|w-z|^{1-\beta}(1+|y|)^{-(1+d)} \\
\text { si } \rho_{0}>1 \text { et } 0<\beta<1 \\
\left|p_{k}(w, y)-p_{k}(z, y)\right| \leq c_{\beta}|w-z|^{1-\beta}(1+|y|)^{-(1+d)} 2^{k \delta\left(1-\beta-\rho_{0}\right)} \\
\text { si } \rho_{0}<1 \text { et } \rho_{0}<1-\beta<1 .
\end{array}\right.
$$

Comme $A_{k}(z)=2^{k(d+1)} A_{0}\left(2^{k} z\right)$, l'équation (B.23) est conséquence de (B.24) et de

$$
\int_{\mathbb{R}^{d}}\left|A_{0}(z)\right||z|^{1-\beta} \mathrm{d} z<\infty \text { pour } 0<\beta<1
$$

Démonstration du lemme 5.1. Le problème étant de nature locale, et puisqu'on sait déjà que $m \in C^{\infty}$, on peut supposer $g\left(\lambda, \lambda^{\prime}\right)=\lambda-\lambda^{\prime}$ et $f_{k}(t, \lambda) \in C_{0}^{1+\rho}\left(R^{2}\right)$ pour $1 \leq k \leq \ell$.

Soit $f_{k}=\sum_{j \geq 0} f_{k, j}$ la décomposition de Littlewood-Paley de $f_{k}$ (avec $f_{k, 0}=\left(S_{0}+\triangle_{0}\right)\left(f_{k}\right)$ ). Posons

$$
v_{k, j}\left(t, \lambda, \lambda^{\prime}\right)=f_{k, j}(t, \lambda)-f_{k, j}\left(t, \lambda^{\prime}\right) .
$$

On a

$$
\sum_{j \geq 0} v_{k, j}=h_{k}=f_{k}(t, \lambda)-f_{k}\left(t, \lambda^{\prime}\right)
$$

Soit $A$ l'ensemble des applications $\alpha$ de $\{1, \ldots \ell\}$ dans $N$. On posera pour $\alpha \in A$

$$
\begin{gathered}
J_{\alpha}=\max \{\alpha(k)\} \\
\sigma(\alpha)=\sum \alpha(k) \\
\beta(\alpha)=\sigma(\alpha)-J_{\alpha}+1
\end{gathered}
$$


On a alors

$$
v^{\alpha}=\prod_{k=1}^{\ell} v_{k, \alpha(k)} .
$$

$$
\prod_{k=1}^{\ell} h_{k}=\sum_{\alpha \in A} v^{\alpha} .
$$

Lemme B.4. Il existe une constante $C_{0}$ telle que :

Les fonctions $\theta_{k, j}\left(t, \lambda, \lambda^{\prime}\right)=\frac{v_{k, j}}{\lambda-\lambda^{\prime}}$ vérifient

$$
\begin{gathered}
\left|\partial^{\gamma} \theta_{k, j}\right|_{L^{\infty}} \leq C_{0} 2^{j|\gamma|} 2^{-j \rho}\left\|f_{k}\right\|_{1+\rho} \\
\left|\int\right| \partial_{\lambda^{\prime}} \theta_{k, j}\left(t, \lambda, \lambda^{\prime}\right)\left|\mathrm{d} \lambda^{\prime}\right|_{L^{\infty}(t, \lambda)} \leq C_{0}(1+j) 2^{-j \rho}\left\|f_{k}\right\|_{1+\rho} .
\end{gathered}
$$

Les fonctions $w^{\alpha}(t, \lambda)=p f \int \frac{v^{\alpha}\left(t, \lambda, \lambda^{\prime}\right)}{\left(\lambda-\lambda^{\prime}\right)^{\ell+1}} \mathrm{~d} \lambda^{\prime}$ vérifient

$$
\begin{gathered}
\left|w^{\alpha}\right|_{L^{\infty}} \leq C_{0}^{\ell} \beta(\alpha) 2^{-\sigma(\alpha) \rho} \prod_{k=1}^{\ell}\left\|f_{k}\right\|_{1+\rho} \\
\left|w^{\alpha}\right|_{L^{\infty}} \leq C_{0}^{\ell} \beta(\alpha) 2^{-\sigma(\alpha) \rho} 2^{J_{\alpha}}\left\|f_{1}\right\|_{\rho} \prod_{k=2}^{\ell}\left\|f_{k}\right\|_{1+\rho} \\
\operatorname{sp}\left(w^{\alpha}\right) \subset\left\{\zeta,|\zeta| \leq 2 c \sum_{k} 2^{\alpha(k)}\right\} .
\end{gathered}
$$

Démonstration. On a pour $j \geq 1, f_{k, j}=\varphi_{j} *_{\lambda} f_{k, j}$ avec $\varphi_{j}=2^{j} \phi\left(2^{j} \lambda\right)$ pour un $\phi$ dans $\mathcal{S}(R)$, d'où

$$
\left\{\begin{array}{l}
\theta_{k, j}=\int \frac{\varphi_{j}(\lambda-z)-\varphi_{j}\left(\lambda^{\prime}-z\right)}{\lambda-\lambda^{\prime}} f_{k, j}(t, z) \mathrm{d} z \\
=\int\left(\int_{0}^{1} \varphi_{j}^{\prime}\left(\lambda+s\left(\lambda-\lambda^{\prime}\right)-z\right) \mathrm{d} s\right) f_{k, j}(t, z) \mathrm{d} z
\end{array}\right.
$$

(B.26) résulte donc de (B.31) et de

$$
\left\|\partial_{\lambda, \lambda^{\prime}}^{\beta} \varphi_{j}^{\prime}\left(\lambda+s\left(\lambda-\lambda^{\prime}\right)-z\right)\right\|_{L^{1}(d z)} \leq C_{0} 2^{j} 2^{j|\beta|}
$$

et pour obtenir (B.27), il suffit décrire $\lambda^{\prime}=\lambda-\mu$ et d'utiliser

$$
\left\{\begin{array}{l}
\int_{|\mu| \leq B}\left|\partial_{\mu} \theta_{k, j}(t, \lambda, \lambda-\mu)\right| \mathrm{d} \mu= \\
\int_{|\mu| \leq B} \mathrm{~d} \mu\left|\int f_{k, j}(t, z) \mathrm{d} z\left(\int_{0}^{1} s \varphi_{j}^{\prime \prime}(\lambda+s \mu-z) \mathrm{d} s\right)\right|= \\
\int_{|\mu| \leq B} \frac{\mathrm{d} \mu}{|\mu|} \mid \int f_{k, j}(t, z) \mathrm{d} z\left(\int_{0}^{1} 2^{2 j}\left(\phi^{\prime}\left(2^{j} \lambda+2^{j} s \mu-2^{j} z\right)-\phi^{\prime}\left(2^{j} \lambda+2^{j} \mu-2^{j} z\right)\right) \mid \mathrm{d} s\right. \\
=2^{j} \int_{|\mu| \leq 2^{j} B} \frac{\mathrm{d} \mu}{|\mu|} \mid \int f_{k, j}\left(t, 2^{-j} z\right) \mathrm{d} z\left(\int_{0}^{1}\left(\phi^{\prime}\left(2^{j} \lambda+s \mu-z\right)-\phi^{\prime}\left(2^{j} \lambda+\mu-z\right)\right) \mid \mathrm{d} s\right.
\end{array}\right.
$$


Pour vérifier (B.28), on choisit pour tout $\alpha$ un $m \in\{1, \ldots, \ell\}$ tel que $\alpha(m)=J_{\alpha}$ et on pose

$$
\Theta^{\alpha}=\prod_{k \neq m} \theta_{k, \alpha(k)} .
$$

On a alors

$$
\left\{\begin{array}{l}
\frac{v^{\alpha}}{\left(\lambda-\lambda^{\prime}\right)^{1+\ell}}=\Theta^{\alpha} \frac{v_{m, \alpha(m)}}{\left(\lambda-\lambda^{\prime}\right)^{2}}=I+I I \\
I=\Theta^{\alpha}(t, \lambda, \lambda) \frac{v_{m, \alpha(m)}\left(t, \lambda, \lambda^{\prime}\right)}{\left(\lambda-\lambda^{\prime}\right)^{2}} \\
I I=\int_{0}^{1} \partial_{\lambda^{\prime}}\left(\Theta^{\alpha}\right)\left(t, \lambda, \lambda+s\left(\lambda-\lambda^{\prime}\right) \theta_{m, \alpha(m)}\left(t, \lambda, \lambda^{\prime}\right) \mathrm{d} s\right.
\end{array}\right.
$$

de sorte que (B.28) résulte de (B.26) et (B.27).

On obtient (B.29) par un argument analogue.

On a

$$
\begin{gathered}
f \frac{1}{g} \frac{h_{1}}{g} \cdots \frac{h_{\ell}}{g} \mathrm{~d} \lambda^{\prime}=\sum_{n} H_{n} \\
H_{n}=\sum_{\alpha \in A_{n}} w^{\alpha} \\
A_{n}=\left\{\alpha \in A: 2^{n-1} \leq \sum_{k} 2^{\alpha(k)}<2^{n}\right\} .
\end{gathered}
$$

On a $s p\left(H_{n}\right) \subset\left\{\zeta ;|\zeta| \leq c 2^{n+1}\right\}$, et comme on a $\rho>0$ (5.4) sera conséquence des estimations

$$
\begin{gathered}
\left\|H_{n}\right\|_{\infty} \leq M^{\ell} 2^{-n \rho} \prod_{k=1}^{\ell}\left\|f_{k}\right\|_{1+\rho} \\
\left\|H_{n}\right\|_{\infty} \leq M^{\ell} 2^{-n \rho} 2^{n}\left\|f_{1}\right\|_{\rho} \prod_{k=2}^{\ell}\left\|f_{k}\right\|_{1+\rho} .
\end{gathered}
$$

Or on vérifie aisément que (B.39) et (B.40) sont conséquences de (B.41) et (B.42) :

$$
\begin{gathered}
\sum_{\alpha \in A_{n}} \beta(\alpha) 2^{-\sigma(\alpha) \rho} \leq C_{1}^{\ell} 2^{-n \rho} \\
\sum_{\alpha \in A_{n}} \beta(\alpha) 2^{-\sigma(\alpha) \rho} 2^{J_{\alpha}} \leq C_{1}^{\ell} 2^{n} 2^{-n \rho}
\end{gathered}
$$

Je remercie L.C. Evans qui m’a signalé que S. Wu a indépendamment obtenu des résultats similaires aux nôtres. 


\section{RÉFÉRENCES}

[1] G. Birkhoff, Helmholtz et Taylor instability. Proc. Symp. Appl. Math XIII. Amer. Math. Soc. (1962) 55-76.

[2] C. Bardos, U. Frisch, C. Sulem et P.L. Sulem, Finite time analyticity for the two and three dimensional Kelvin-Helmholtz instability. CMP 80 (1981) 485-516.

[3] J.-M. Bony, Calcul symbolique et propagation des singularités pour les équations aux dérivées partielles non linéaires. Ann. Sci. Ec. Norm. Sup. IV 14 (1981) 209-246.

[4] J.-M. Delort, Existence de nappes de tourbillon en dimension deux. J. Amer. Math. Soc. 4 (1991) 553-586.

[5] J. Duchon et R. Robert, Global vortex sheet solutions of Euler equation in the plane. J. Differential Equations 73 (1988) 215-224.

[6] G. Lebeau, Régularité du problème de Kelvin-Helmholtz pour l'équation d'Euler 2d. Séminaire X-EDP 2000/2001, exposé 1 (2000). 